ARTICLE

\title{
Translation and codon usage regulate Argonaute slicer activity to trigger small RNA biogenesis
}

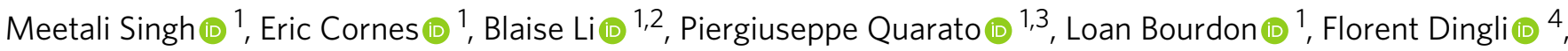
Damarys Loew (iD ${ }^{4}$, Simone Proccacia (i) ${ }^{1,5} \&$ Germano Cecere (iD) ${ }^{1 \times}$

In the Caenorhabditis elegans germline, thousands of mRNAs are concomitantly expressed with antisense 22G-RNAs, which are loaded into the Argonaute CSR-1. Despite their essential functions for animal fertility and embryonic development, how CSR-1 22G-RNAs are produced remains unknown. Here, we show that CSR-1 slicer activity is primarily involved in triggering the synthesis of small RNAs on the coding sequences of germline mRNAs and post-transcriptionally regulates a fraction of targets. CSR-1-cleaved mRNAs prime the RNAdependent RNA polymerase, EGO-1, to synthesize 22G-RNAs in phase with translating ribosomes, in contrast to other 22G-RNAs mostly synthesized in germ granules. Moreover, codon optimality and efficient translation antagonize CSR-1 slicing and 22G-RNAs biogenesis. We propose that codon usage differences encoded into mRNA sequences might be a conserved strategy in eukaryotes to regulate small RNA biogenesis and Argonaute targeting.

\footnotetext{
${ }^{1}$ Mechanisms of Epigenetic Inheritance, Department of Developmental and Stem Cell Biology, Institut Pasteur, UMR3738, CNRS, Paris, France. ${ }^{2}$ Hub de Bioinformatique et Biostatistique-Département Biologie Computationnelle, Institut Pasteur, Paris, France. ${ }^{3}$ Sorbonne Université, Collège Doctoral, Paris, France. ${ }^{4}$ Institut Curie, PSL Research University, Centre de Recherche, Laboratoire de Spectrométrie de Masse Protéomique, Paris, France. ${ }^{5}$ Università di Trento, Trento TN, Italy. ${ }^{凶}$ email: germano.cecere@pasteur.fr
} 
$\mathrm{n}$ animals, small RNAs expressed in the germline and transmitted to the embryo act as a defense mechanism to repress foreign RNAs such as viruses, transposons, and other repetitive elements (REs). These small RNAs are essential for fertility and genome integrity ${ }^{1,2}$. Their function is controlled by the conserved family of Argonaute proteins (AGOs), which loads the small RNAs and functions to repress complementary messenger RNA (mRNA) targets through their endonuclease activity or by recruiting other effector silencing proteins ${ }^{3-6}$. The C. elegans germline contains a complex small RNA regulatory network, with different classes of small RNAs, multiple AGO effectors, and diverse biogenesis pathways ${ }^{7}$. One of the most abundant classes of endogenous small RNAs in the germline is the 22G-RNAs, which are single-stranded antisense small RNAs produced by RNA-dependent RNA polymerase (RdRPs) as part of an amplification system to silence target transcripts (reviewed in ${ }^{7}$ ). The production of 22G-RNAs targeting REs is triggered by over 15,000 PIWI-interacting RNAs (piRNAs or 21U-RNAs) and loaded by Worm-specific Argonautes (WAGOs) to silence REs, including the nuclear Argonaute HRDE-1 ${ }^{8-12}$. 22G-RNAs are also produced from most germline-expressed mRNAs by the RdRP EGO-1 and loaded into the Argonaute CSR-1 ${ }^{13,14}$. In contrast to the 22G-RNAs antisense to REs, which can be triggered in response to piRNAs, the primary trigger for generating CSR-1 22G-RNAs and why many germline mRNAs become targeted by CSR-1 is still unknown (Supplementary Fig. 1).

Given that the C. elegans piRNAs can trigger their targets' silencing by imperfect complementarity, and therefore potentially target germline-expressed mRNAs ${ }^{15-17}$, the targeting by CSR-1 22G-RNAs can function as an anti-silencing mechanism to protect germline mRNAs from piRNAs silencing ${ }^{14,18,19}$. The antisilencing function of CSR-1 can occur in the nucleus or P granules. In the nucleus, CSR-1 has been shown to interact with chromatin in a 22G-RNA-dependent manner ${ }^{14}$ where it can counteract piRNA-mediated silencing by antagonizing the binding of the nuclear Argonaute protein HRDE-1 to nascent germline transcripts $^{20,21}$. In P granules, CSR-1 can scan the mRNAs exiting the nuclear pore and compete with piRNA targeting ${ }^{15}$. The anti-silencing function of CSR-1 was primarily established with single-copy transgenes ${ }^{16,18,19}$. However, germline mRNAs remain protected from piRNAs silencing even in the absence of CSR-1 $1^{17}$, and sequence-encoded features of germline mRNAs have also been proposed to prevent piRNA silencing ${ }^{15,17}$. To what extent endogenous germline-expressed genes are regulated by CSR-1 and piRNA pathways' antagonistic functions remain elusive (Supplementary Fig. 1).

In addition, CSR-1 has been proposed to regulate the expression of its germline targets directly. Transcriptomic analyses of CSR-1 loss of function alleles have shown that CSR-1 promotes the expression of its target genes in hermaphrodites and males ${ }^{14,22,23}$. On the other hand, of the Argonautes that load 22G-RNAs, only CSR-1 has demonstrated slicer activity on target mRNA in vitro ${ }^{24}$, and worms expressing a CSR-1 catalytic mutant protein show upregulation of its germline target genes ${ }^{25}$. Thus, it remains unclear whether CSR-1 positively or negatively regulates the expression of its target mRNAs. This is because all these studies have been performed using different methodologies at different developmental stages using either CSR-1 mutants, hypomorphs, or CSR-1 KO rescued with transgenic CSR-1 catalytic mutant ${ }^{14,22,23,25}$. As a result, the gene expression changes observed in the different studies do not largely overlap (Supplementary Fig. $2 a, b)^{14,23,25}$. Therefore, the gene regulatory functions of germline CSR-1 22G-RNAs remain incompletely understood (Supplementary Fig. 1).

Similarly, the biogenesis of CSR-1 22G-RNAs remains mysterious. Many germline Argonautes, including CSR-1 and PIWI, and proteins involved in 22G-RNA biogenesis, including RdRPs, localize to germ granules ${ }^{14,26}$. These germ granules are thought to be the site for the biogenesis of all germline 22G-RNAs. Germ granules are organized in sub-compartments- $\mathrm{M}, \mathrm{Z}$, and $\mathrm{P}$ granules ${ }^{27}$. Disruption of $M$ granule (also known as mutator foci), which participates in piRNA-dependent 22G-RNA production, has no apparent effect on CSR-1 22G-RNAs ${ }^{28,29}$. Moreover, the type of RNA template used by the EGO-1 RdRP to generate CSR1 22G-RNAs remains mysterious. During exogenous RNAi, the addition of alternating non-templated uridine (U) and guanosine $(\mathrm{G})$ ribonucleotides (polyUG) to the $3^{\prime}$ termini of cleaved mRNA targets by RDE- 3 recruits RdRPs EGO- 1 and RRF- 1 to synthesize $22 \mathrm{G}-\mathrm{RNAs}^{30,31}$. However, RDE-3 is not required to generate CSR-1 22G-RNAs ${ }^{26,31}$. Thus, the subcellular location and RNA substrate used to create 22G-RNAs is unknown.

In the current study, we elucidate CSR-1 catalytic activitydependent and independent germline gene regulation and decipher the rules governing CSR-1 22G-RNA biogenesis. We demonstrate that the slicer activity of CSR-1 triggers the biogenesis of 22G-RNAs antisense to the coding sequence of germline mRNAs. We establish that CSR-1 22G-RNAs are synthesized on an actively translated mRNA template in the cytosol, independent of germ granules. Overall, this study establishes that translation and codon usage dictate CSR-1 slicer activity on a target mRNA to regulate small RNA biogenesis and functions.

\section{Results}

Defects in CSR-1 catalytic activity mainly impact 22G-RNA abundance. Both csr-1 catalytic mutant (csr-1 ADH) and knockout $(c s r-1 \mathrm{KO})$ worms show reduced fertility and $100 \%$ embryonic lethality ${ }^{32}$. However, their gene expression profiles are different (Supplementary Fig. 2a, b). We hypothesized that the global impact of CSR-1 mutations on gene expression might depend on the developmental context and might be biased by developmental defects ${ }^{14,33}$. Indeed, we observed differences during oogenesis in $c s r-1 \mathrm{ADH}$ and $c s r-1 \mathrm{KO}$ worms marked by a delayed onset of oocyte production and increased accumulation of oocytes in the germline in $c s r-1 \mathrm{ADH}$ at a more advanced age compared to wild-type (WT) (Supplementary Fig. 2c-f). To overcome this limitation, we developed a sorting strategy to obtain a synchronized population of WT and first-generation homozygotes for csr-1 KO or csr-1 ADH strains using COPAS biosorter, which allowed us to collect almost a pure population of $\mathrm{M}+/ \mathrm{Z}$ - mutants (Supplementary Fig. 2g). Using this strategy, we enriched for larval stage late L4 worms, characterized by a closed vulva and absence of oocytes and lacking the germline developmental abnormality (Supplementary Fig. 2h, i).

Next, to precisely evaluate the role of CSR-1, we measured small RNA accumulation (sRNA-seq), transcription (GRO-seq), mRNA stability (RNA-seq), and translation (Ribo-seq) in WT and mutant worms. In addition, to assess the direct effect of CSR$122 \mathrm{G}-\mathrm{RNAs}$ on these processes, we sequenced the small RNAs bound to immunoprecipitated CSR-1 from similarly sorted late L4 worms to precisely identify the CSR-1 targets at the same developmental stage. We detected a total of 4803 genes with antisense 22G-RNAs loaded into CSR-1 (IP over input $\geq$ twofold enrichment and $R P M \geq 1$ in each replicate of CSR-1 IP) (Supplementary Data 1). These mRNA targets are germline enriched and largely overlap with previously defined targets ${ }^{14,22}$ with some variations based on developmental stages studied (Supplementary Fig. 3a, b). The csr-1 ADH displayed a global loss of 22G-RNAs for the majority of CSR-1 targets (Fig. 1a, c). However, only $7.7 \%(n=119)$ of CSR-1 targets with $>2$-fold reduction of 22G-RNAs $(n=1536)$ showed increased mRNA levels, and only one showed twofold downregulation (Fig. 1b), 

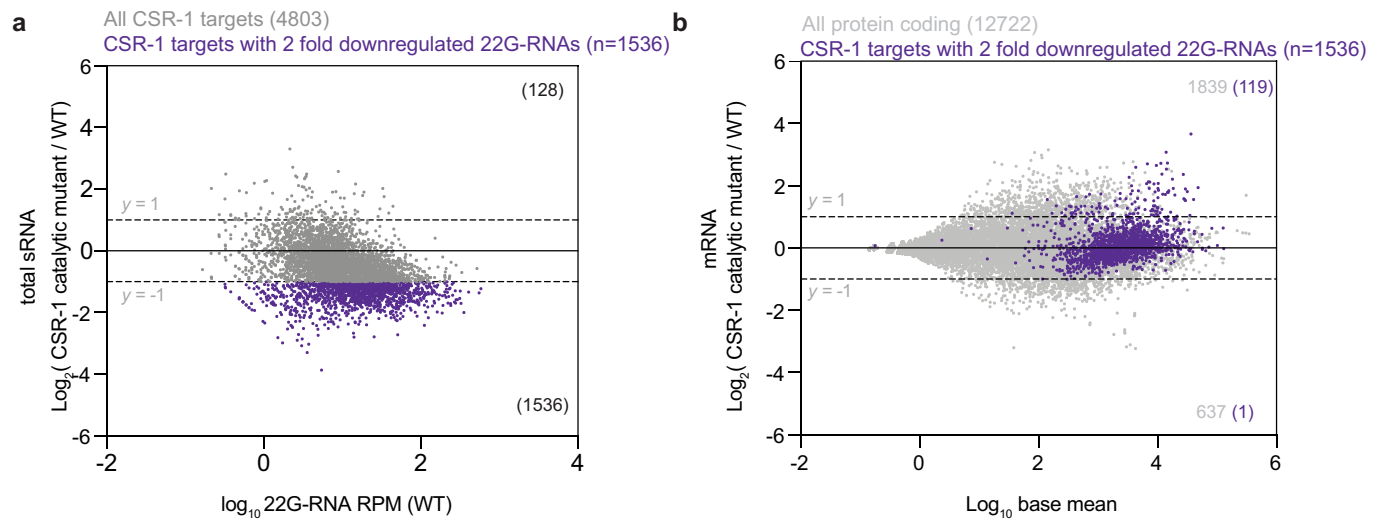

C
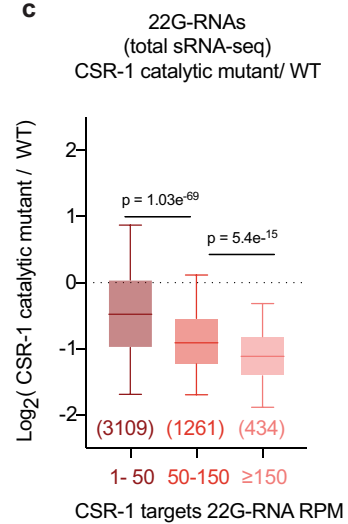

CSR-1 targets 22G-RNA RPM d
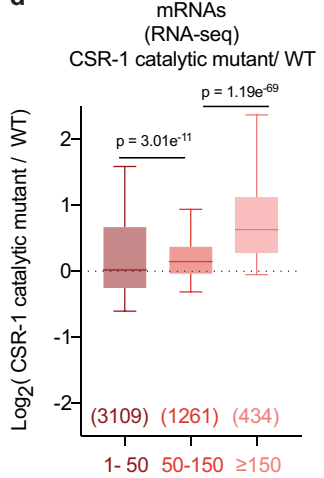

CSR-1 targets 22G-RNA RPM e

$$
\begin{aligned}
& \text { Translation } \\
& \text { (Ribo-seq) }
\end{aligned}
$$

CSR-1 catalytic mutant/ WT

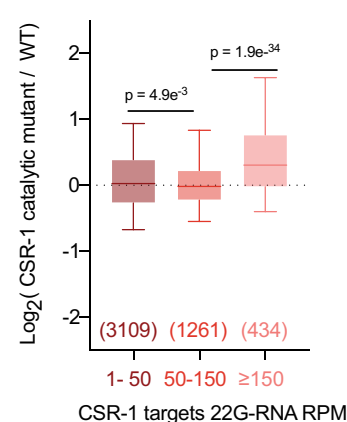

f

Transcription
(GRO-seq)
CSR-1 catalytic mutant/ WT

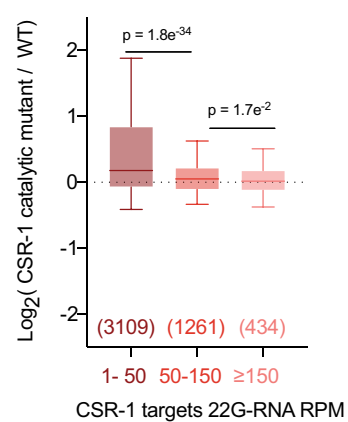

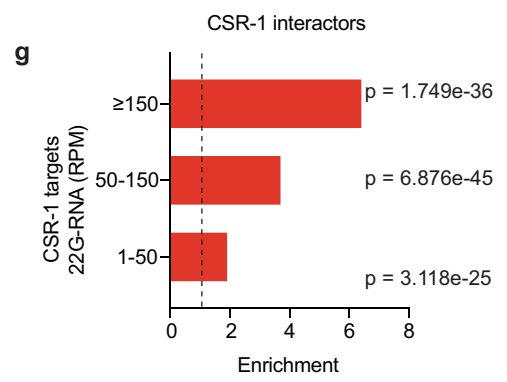

h

CSR-1 slicer independent protected genes $(n=227)$

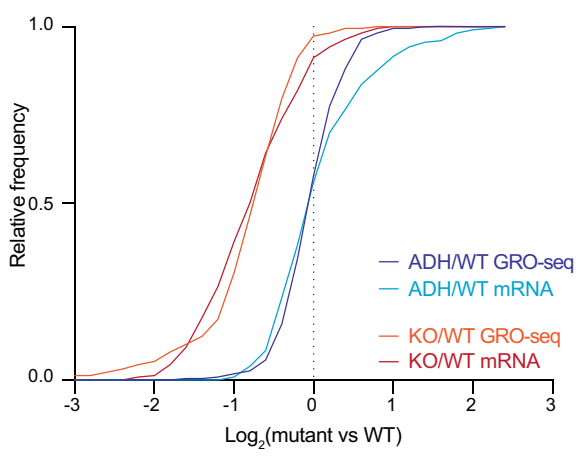

22G-RNAs

i (HRDE-1 IP-sRNA-seq )

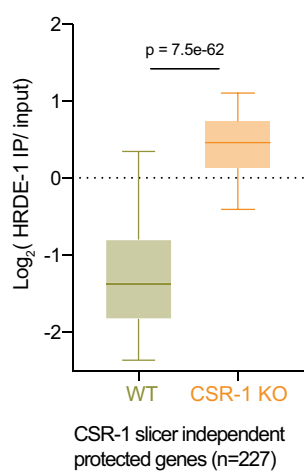

Fig. 1 Defects in CSR-1 catalytic activity mainly impacts 22G-RNA abundance. a MA-plot showing total 22G-RNA log 2 fold-change for CSR-1 ADH (catalytic mutant) compared to WT. The number in parenthesis indicates the number of misregulated genes $\geq 2$-fold. The average from two biological replicates is shown. b MA-plot showing mRNA $\log _{2}$ fold-change for CSR-1 ADH compared to WT. Genes with 22G-RNAs with twofold downregulation in CSR-1 ADH compared to WT are highlighted in purple. The average from two biological replicates is shown, with "base mean" computed using DESeq2 ${ }^{89}$. The number in parenthesis indicates the number of misregulated genes $\geq 2$-fold (gray dots-all protein-coding genes, purple- CSR-1 targets with twofold downregulated 22G-RNAs). c-f Box plots showing the $\log _{2}$ fold-change in CSR-1 ADH compared to WT strain for total 22G-RNAs (sRNA-seq) (2 biological replicates) (c); or mRNAs (RNA-seq) (2 biologically replicates) (d); mRNAs engaged in translation (Ribo-seq) (3 biological replicates) (e); and nascent RNAs (GRO-seq) (2 biologically replicates) (f), The distribution for the CSR-1 targets with 22G-RNA in CSR-1 IP with 1-50 RPM, 50-150 RPM, or $\geq 150$ RPM is shown (gene list in Supplementary Data 1). $\mathbf{g}$ Enrichment of CSR-1 interactors in different CSR-1 targets categories based on 22G-RNA abundance. The dashed line at 1 indicates no enrichment. P-values were calculated by Exact hypergeometric probability using an automated tool available at http:// nemates.org/MA/progs/overlap_stats.html. h Cumulative frequency distribution for CSR-1 slicer-independent protected targets (downregulated in CSR-1 $\mathrm{KO}$ compared to the CSR-1 ADH in GRO-seq, gene list in Supplementary Data 1). The comparison shows GRO-seq $\left(P=1.6 \mathrm{e}^{-49}\right)$ and RNA-seq $\left(P=4.2 e^{-37}\right)$ for CSR-1 KO or CSR-1 ADH compared to WT. i Box plots showing the $\log _{2}$ fold-change of 22G-RNAs (sRNA-seq) in HRDE-1 IPs compared to input in WT, CSR-1 KO strains. Data is representative of two biological replicates. For all the box plots, the line indicates the median value, the box indicates the first and third quartiles, and the whiskers indicate the 5th and 95th percentiles, excluding outliers. Two-tailed $P$-values were calculated using Mann-Whitney-Wilcoxon tests. The sample size $\mathrm{n}$ (genes) is indicated in parentheses. Source data are provided as a Source Data file. 
indicating that most mRNA targets are not destabilized by CSR slicer activity. We also detected some targets with upregulated levels of 22G-RNAs (Fig. 1a), which belong to spermatogenic genes and are being investigated in an independent study. We further divided CSR-1 targets into three bins based on 22G-RNA amounts loaded by CSR-1 in IP and analyzed gene expression changes and dependence on 22G-RNA levels. The increase in mRNA and translational levels of the targets in csr-1 $\mathrm{ADH}$ correlated with 22G-RNA levels in CSR-1 IPs in a dosedependent manner (Fig. 1d, e) in agreement with a previous report $^{25}$, but their transcription was unaffected (Fig. 1f). Therefore, our results support a previously developed model that CSR-1 slices a subset of mRNA targets having abundant 22G-RNAs at the post-transcriptional level ${ }^{25,32}$. Moreover, CSR-1 interactors identified by mass spectrometry (MS/MS) are enriched with CSR1 targets that are post-transcriptionally regulated by CSR-1 (Fig. 1g and Supplementary Fig. 3c). Most of these targets are direct interactors and are not impacted by RNase treatment (Supplementary Fig. 3d-f). Thus, CSR-1 slicer activity negatively regulates the expression of its own interactors, including CSR-1, suggesting a negative feedback loop.

Overall, these results suggest that the main role of CSR-1 catalytic activity is to control the accumulation of CSR-1 interacting 22G-RNAs. In addition, CSR-1 post-transcriptionally regulates a small fraction of CSR-1 targets that have highly abundant 22G-RNAs.

CSR-1 protects a subset of oogenic enriched targets from piRNA-mediated transcriptional silencing. Similar to $c s r-1$ $\mathrm{ADH}$ worms, $c s r-1 \mathrm{KO}$ worms displayed a loss of $22 \mathrm{G}$-RNAs as well as an upregulation of a subset of target mRNAs characterized by a high abundance of 22G-RNAs (Supplementary Fig. 4a-d). However, the level of upregulation of CSR-1 target mRNAs was significantly lower in the csr- $1 \mathrm{KO}$ compared to the $c s r-1 \mathrm{ADH}$, possibly due to decreased transcription (Supplementary Fig. 4e). Indeed, we found that a subset of target genes displayed downregulated transcription and reduced mRNA levels in the $\mathrm{KO}$ compared to $c s r-1 \mathrm{ADH}$. These were downregulated in $\mathrm{KO}$ compared to WT but were unaffected in the $c s r-1 \mathrm{ADH}$ (Fig. 1h). The majority of these genes (53\%) were enriched for oogenic mRNAs (see Supplementary Data 1 for gene list) (Supplementary Fig. 4f), and there was no clear correlation with the abundance of 22G-RNAs loaded by CSR-1 for these targets. Given that CSR-1 is proposed to protect germline transcripts from piRNA-mediated silencing, we hypothesized that in the $c s r-1 \mathrm{KO}$, piRNAs can trigger the loading of 22G-RNAs into the nuclear Argonaute HRDE-1 resulting in the reduced transcription of this subset of CSR-1 targets. We observed an increased number of CSR-1 targets with their 22G-RNAs being loaded by HRDE-1 in csr-1 KO compared to WT (Supplementary Fig. 4g). Indeed, we noticed HRDE-1 loads increased levels of 22G-RNAs from transcriptionally downregulated CSR-1 targets in the csr-1 KO (Fig. 1i). These experiments provide evidence that endogenous genes can be targeted by HRDE-1 in the absence of CSR-1, supporting its anti-silencing role. We further show that CSR-1 sliced targets and CSR-1 protected targets are mutually exclusive (Supplementary Fig. 4h), highlighting a slicer-dependent regulation of gene expression and slicer-independent role in protecting a subset of oogenic targets from piRNA-mediated HRDE-1 transcriptional silencing.

CSR-1 catalytic activity is required for biogenesis of 22G-RNAs antisense to the coding sequence of target mRNAs. The global reduction of CSR-1-bound 22G-RNAs observed in CSR-1 mutants, including CSR-1 sliced as well as CSR-1 protected targets (Supplementary Fig. 4i), suggests that CSR-1 catalytic activity is required for 22G-RNA loading or biogenesis. Despite the reduction in total 22G-RNAs in the csr-1 ADH strain, an enrichment of 22G-RNAs in IP over input was observed for CSR$1 \mathrm{ADH}$ protein (Supplementary Fig. 5a, b), suggesting that catalytic inactive CSR-1 (CSR-1 ADH) can still bind the 22G-RNAs produced in the mutant. In fact, CSR-1 ADH showed enhanced binding efficiency compared to WT CSR-1, suggesting that either the loading of 22G-RNA is more efficient in CSR-1 ADH or the catalytic mutant protein stabilizes its interacting 22G-RNAs.

We then investigated the distribution of CSR-1-bound 22GRNAs along the target-gene bodies. We found that the reduction in 22G-RNAs in csr-1 ADH and KO primarily occurred antisense to the coding sequence (CDS) of CSR-1 targets, whereas 22GRNAs derived from the 3'-untranslated region (3'UTR) were largely unaffected (Fig. 2a-e and Supplementary Fig. 5c). These results indicate that the RdRP fails to synthesize 22G-RNAs on the CDS in the absence of catalytic activity.

The RdRP EGO-1 has been proposed to exclusively synthesize CSR-1-bound 22G-RNAs ${ }^{13,14,26}$. We confirmed these results by using an ego-1 knockout (KO) and sequenced 22G-RNAs, which were depleted both at CDS and 3 UTR (Fig. 2d, f). To understand whether the small RNAs produced on the 3 UTR in the absence of CSR-1 protein or its catalytic activity are also synthesized by EGO-1, we efficiently depleted CSR-1 using an auxin-induced degradation system, combined with ego-1 knockdown by RNAi (Supplementary Fig. 5d-f). First, we confirmed that CSR-1 22GRNAs were depleted on CDS and enriched on 3'UTR upon auxininduced CSR-1 depletion (Fig. 2g, h). Next, we observed reduced 22G-RNAs from both CDS as well as 3'UTR upon ego-1 knockdown by RNAi (Fig. 2g, i and Supplementary Fig. 5g, h), implying that EGO-1 may be exclusively responsible for the synthesis of the CSR-1 22G-RNAs in both WT and the csr-1 mutants. However, the catalytic activity of CSR-1 is required to efficiently generate EGO-1-dependent 22G-RNAs along the coding sequences of target mRNAs. To understand if another class of endogenous small RNAs, the 26G-RNA ${ }^{34,35}$, may be priming the EGO-1 recruitment at the 3 'UTR, we combined ego-1 RNAi with mutant of RdRP, $r r f-3-/-$, which is responsible for 26G-RNA production ${ }^{34,35}$. However, we did not observe any contribution of RRF-3 produced 26G-RNAs in the biogenesis of CSR-1 22G-RNAs and EGO-1 priming on 3'UTR of CSR-1 targets (Supplementary Fig. 6a-c). Also, we did not observe any compositional bias for 22-nt small RNAs derived from CDS and 3'UTR (Supplementary Fig. 6d, e). CSR-1 -associated 22G-RNAs can also be poly uridylated $(\mathrm{U})^{36}$. Thus, we investigated whether there was any difference in the levels of CSR-1 22G-RNAs poly (U) in both $c s r-1 \mathrm{ADH}$ and $c s r-1 \mathrm{KO}$ compared to WT. Our analysis showed a loss of CSR-1-associated 22G-RNAs poly (U) both at CDS and 3'UTR (Supplementary Fig. 6f-h), similar to what we have observed for total CSR-1 22G-RNAs. The CSR-1 22G-RNAs poly(U) were also globally reduced in ego-1 $\mathrm{KO}$ (Supplementary Fig. 6f, g), suggesting poly(U) addition happens post-22G-RNA biogenesis ${ }^{36}$. Thus, how EGO-1 is recruited at 3'UTR remains to be investigated.

Finally, we tested whether the restored expression of CSR-1 is sufficient to generate EGO-1-dependent 22G-RNAs on the gene body. For this purpose, we depleted CSR-1 by auxin-induced degradation for $38 \mathrm{~h}$ after hatching ( $0 \mathrm{~h}$ recovery) and then reintroduced CSR-1 by recovering expression for 5 and $10 \mathrm{~h}$ (Supplementary Fig. 6i). As expected, the depletion of CSR-1 caused a loss of $22 \mathrm{G}$-RNA accumulation on the CDS (Fig. $2 \mathrm{j}$ and Supplementary Fig. $6 \mathrm{i}-$ see $0 \mathrm{~h}$ recovery). However, upon reintroduction of CSR-1 expression (5 and $10 \mathrm{~h}$ recovery), we observed a steady increase of 22G-RNAs, mainly on the CDS (Fig. 2j, k). The lack of complete recovery of 22G-RNAs could be 
a

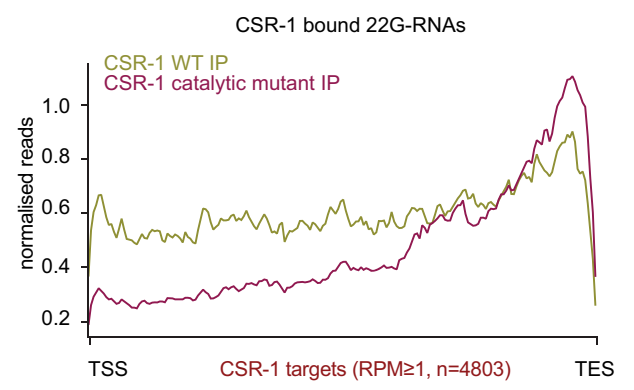

d

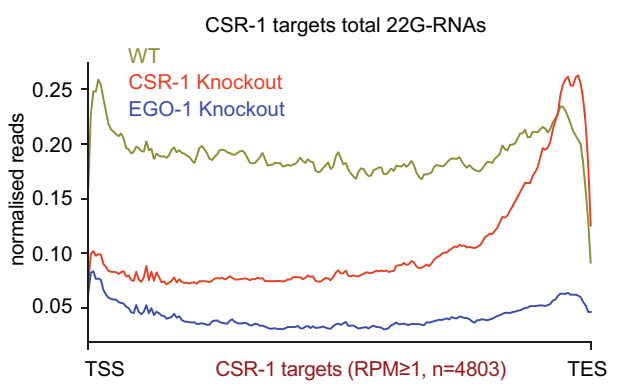

g

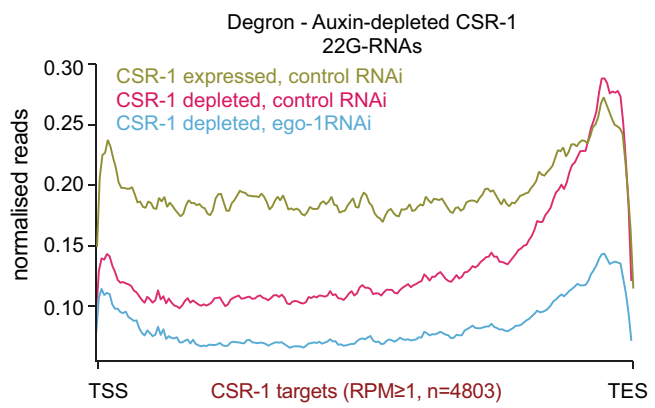

j

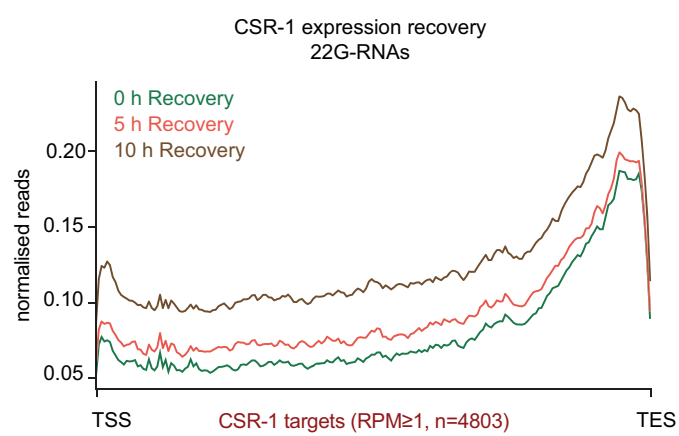

due to the accumulation of germline defects as a result of CSR-1 depletion during the initial period of germline development.

Overall, these data demonstrate that EGO-1 can be recruited on the 3'UTR of target mRNAs and initiate the production of 22G-RNAs. However, CSR-1-mediated slicing of mRNAs is required to template the production of small RNAs on the gene body.
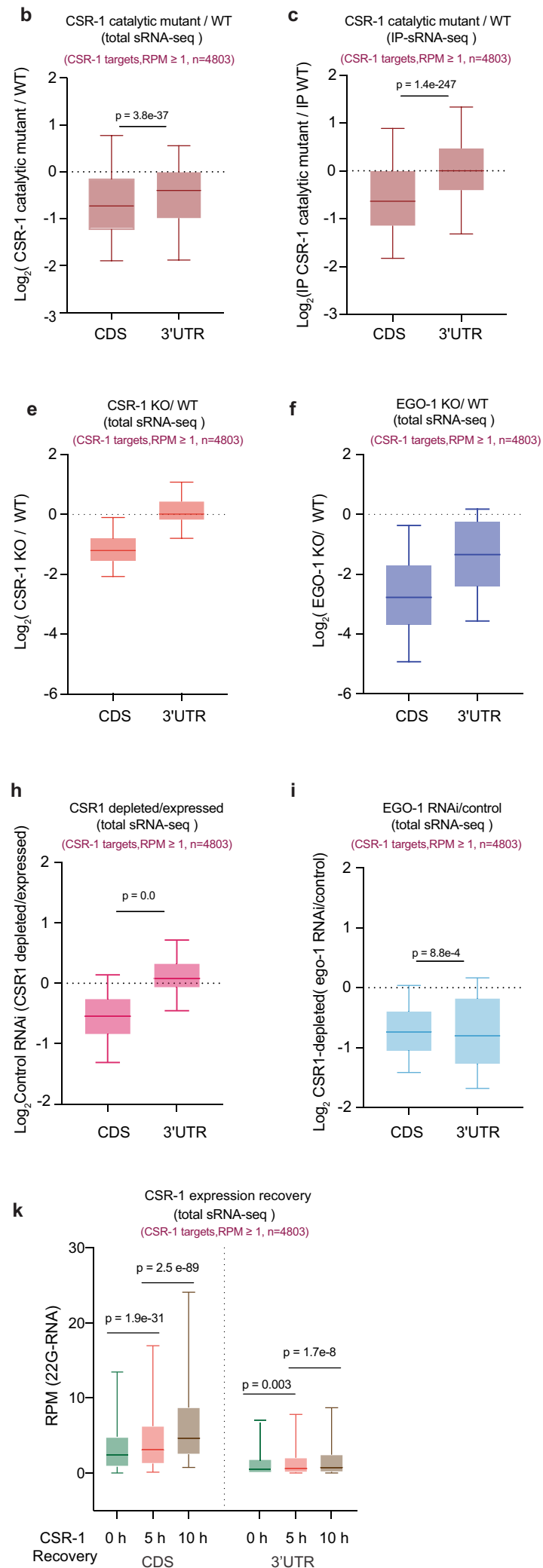

Biogenesis of CSR-1 22G-RNAs and the regulation of their targets occurs in the cytosol. PIWI and RNAi biogenesis factors are known to localize in perinuclear condensates, called germ granules, and these germ granules have been proposed to be the site for biogenesis of $22 \mathrm{G}-\mathrm{RNAs} \mathrm{s}^{28,37-39}$. Germ granules have been shown to be organized in different compartments, namely P, M (also known as mutator foci), and Z granules ${ }^{27}$. CSR-1 and 
Fig. $\mathbf{2}$ CSR-1 catalytic activity is required for the biogenesis of 22G-RNAs antisense to the coding sequence. a, $\mathbf{d}, \mathbf{g}$, $\mathbf{j}$ Metaprofile analysis showing the distribution of normalized 22G-RNA (sRNA-seq) reads (RPM) along all CSR-1 targets ( $\geq 1$ RPM, $n=4803$ ) in WT CSR-1 or CSR-1 ADH immunoprecipitation (IP) (a); in WT, cSr-1 KO and ego-1 KO (d); upon ego-1 RNAi and Control RNAi treated in Auxin-depleted CSR-1 degron background (CSR-1 depleted) and degron control (CSR-1 expressed) (g); after depletion of CSR-1, in the CSR-1 degron strain for $38 \mathrm{~h}$ by growing on auxin containing plates and recovery of CSR-1 expression by transferring on plates without auxin for 0,5 , and $10 \mathrm{~h}(\mathbf{j})$, TSS indicates the transcriptional start site, TES indicates the transcriptional termination site. An average of two biological replicates is shown. $\mathbf{b}, \mathbf{c}$ Box plots showing the $\log _{2}$ fold-change of the amount of total 22G-RNA generated from CDS and 3UTR of all CSR-1 targets in CSR-1 ADH compared to WT (b); 22G-RNA generated from CDS and 3UTR of all CSR-1 targets bound in CSR-1 ADH IP compared to WT CSR-1 IP (c). e, f Box-plot showing the $\log _{2}$ fold-change in the amount of 22G-RNA generated from CDS and 3'UTR of all CSR-1 targets in csr-1 KO compared to WT (e) and in ego-1 KO compared to WT (f). $\mathbf{h}, \mathbf{i}$ Box-plot showing the log 2 fold-change in the amount of 22G-RNA generated from CDS and 3'UTR of all CSR-1 targets in Auxin-depleted CSR-1 compared to non-depleted CSR-1 degron control in control RNAi background (h); for ego-1 RNAi compared to control RNAi treated in Auxin-depleted CSR-1 degron background (i). k Box-plot representing the data in $\mathbf{j}$ showing the RPM of 22G-RNAs generated from CDS and 3'UTR of CSR-1 targets (22G-RNA $\geq 1$ RPM) for CSR-1 expression recovered for 0, 5, or $10 \mathrm{~h}$. For all the box plots, the line indicates the median value, the box indicates the first and third quartiles, and the whiskers indicate the 5th and 95th percentiles, excluding outliers. Two-tailed $P$-values were calculated using Mann-Whitney-Wilcoxon tests. For all the experiments, the sample size $n$ (genes) is indicated in parentheses, which include all CSR-1 targets. For all experiments, data is representative of two biological replicates. Source data are provided as a Source Data file.

EGO-1 localize in both cytosol and the $\mathrm{P}$ granules ${ }^{14}$, suggesting that the biogenesis of CSR-1 22G-RNAs might also occur in these organelles. To test this possibility, we used RNAi to simultaneously deplete four core components of P granules ( $p g l-1, p g l-3$, glh-1, and glh-4) $)^{40}$, (Supplementary Fig. 7a). This treatment was sufficient to disrupt not only $\mathrm{P}$ granules but also mutator foci and $\mathrm{Z}$ granules as observed by imaging of their respective components PGL-1 and DEPS-1 (P granule), MUT-16 (mutator foci), and ZNFX-1 (Z granule) (Fig. 3a). Mutator foci were previously not shown to be disrupted by RNAi against either single or two components of $\mathrm{P}$ granule ${ }^{28}$. However, RNAi against four $\mathrm{P}$ granule components disrupts mutator foci also (Fig. 3a). The treatment also depleted the majority of CSR-1 localization in $\mathrm{P}$ granules (Fig. 3b). However, the cytosolic localization of CSR-1 remained unaffected (Fig. 3b). We still observed a residual granular localization of CSR-1, which we attribute to a lack of $100 \%$ knockdown during RNAi treatment. In fact, remaining CSR-1 localized with residual DEPS-1 (a component of P granule) upon P granule RNAi (Fig. 3b). Z granule mutant $(z n f x-1)$ or mutator foci mutant (mut-16) did not affect CSR-1 localization to $\mathrm{P}$ granule (Supplementary Fig. S7b).

Next, we evaluated the effects of loss of germ granules on $22 \mathrm{G}$ RNA biogenesis. Though piRNA-dependent 22G-RNAs were globally depleted upon P granule RNAi treatment (Fig. 3c), CSR1 22G-RNAs were unaffected upon $\mathrm{P}$ granule RNAi treatment, despite the loss of perinuclear CSR-1 P granule localization (Fig. 3b, c). Furthermore, CSR-1 targets were not upregulated upon P granule RNAi (RNA-seq data from ${ }^{41}$ ) (Fig. $3 \mathrm{~d}$ and Supplementary Fig. 7a). Though a synthesis of CSR-1 22G-RNAs in $\mathrm{P}$ granules cannot be completely ruled out, these results highlight that majority of CSR-1 22G-RNA biogenesis occurs in the cytosol, and $\mathrm{P}$ granules are dispensable.

Translating mRNAs serve as the template for 22G-RNA biogenesis. Our data so far suggest that majority of CSR-1 22G-RNAs might be generated in the cytosol. Consistent with CSR-1 localization in the cytosol and $\mathrm{P}$ granules, we identified ribosomal and ribosomal-associated proteins, which are enriched in the cytosol, and germ granule components in our immunoprecipitation-mass spectrometry (IP-MS/MS) as direct CSR-1 interactors (Fig. 4a). Ribosomal interactors of CSR-1 were not lost upon RNase treatment, contrary to ribosomal interactors of PIWI, which are lost on RNase treatment (Supplementary Fig. 7c), suggesting that CSR-1 directly interacts with ribosomal proteins. Moreover, CSR-1 ADH showed reduced co-purification of ribosomal proteins and increased co-purification of $\mathrm{P}$ granule components, compared to CSR-1 WT (Fig. 4b). The catalytic mutation leads to an enriched localization of CSR-1 ADH within P granules, as can be seen with co-localization with GLH-1 (a component of $\mathrm{P}$ granule) in enlarged granules and this increased expression is consistent with the observation that CSR-1 self-regulates its expression (Fig. 4c).

Based on these data, we hypothesized that 22G-RNAs are synthesized in the cytosol, using translating mRNAs as templates. To test this hypothesis, we performed polysome profile and immunoblot for CSR-1 and EGO-1, which were both enriched in polysome fractions, suggesting they interact with translating mRNAs (Fig. 4d). In contrast, PIWI and PGL-1 (a P granule component) were not enriched in the polysome fractions, further supporting the synthesis of PIWI-dependent 22G-RNAs in P granules, which are devoid of mRNAs engaged in translation ${ }^{42}$.

We then mapped the distance between the start of the 29nucleotide Ribosomal Protected Fragments (RPF) ${ }^{43}$ and the $5^{\prime}$-end of CSR-1 22G-RNAs (Supplementary Fig. 7d). We observed the characteristic three-nucleotide (3-nt) periodicity pattern typical of ribosomal footprints (Fig. 4e), indicating that the synthesis of CSR-1 22G-RNAs occurs on mRNA templates engaged in translation in phase with the ribosome. In contrast, the HRDE-1 loaded 22GRNAs of P granule-dependent piRNA targets (Supplementary Data 1) did not show phasing with ribosomes as observed due to a lack of three-nucleotide periodicity and were randomly distributed (Fig. 4e and Supplementary Fig. 7d), in agreement with the fact that $\mathrm{P}$ granules are devoid of translating mRNAs ${ }^{42,44}$ and PIWI is not enriched in polysome fractions. Altogether these results suggest that CSR-1 cleaves actively translating mRNAs, which become the template for EGO-1-mediated synthesis of 22G-RNAs on the coding sequence of mRNA targets.

mRNA translation antagonizes CSR-1 22G-RNA biogenesis. EGO-1-mediated synthesis of CSR-1 22G-RNAs does not occur on every germline mRNA at similar levels, and we found that the levels of 22G-RNA are independent of the levels of the mRNA template (Supplementary Fig. 8a). Given our observations that, actively translating mRNAs serve as the template for CSR-1 22GRNAs, we hypothesized that the translation efficiency (TE) of germline mRNAs impacts CSR-1 22G-RNA biogenesis. To test this hypothesis, we calculated the TE of CSR-1 targets using the Ribo-seq and RNA-seq data from WT worms at the late L4 stage. We observed that levels of CSR-1-associated 22G-RNAs produced from a target mRNA were inversely correlated with their TE (Fig. 5a), suggesting that translation antagonizes the biogenesis of CSR-1 22G-RNAs.

Codon usage and the availability of the tRNA pool influence $\mathrm{TE}^{45,46}$. Therefore, we investigated whether these mechanisms affect the biogenesis of CSR-1 22G-RNAs. We determined 


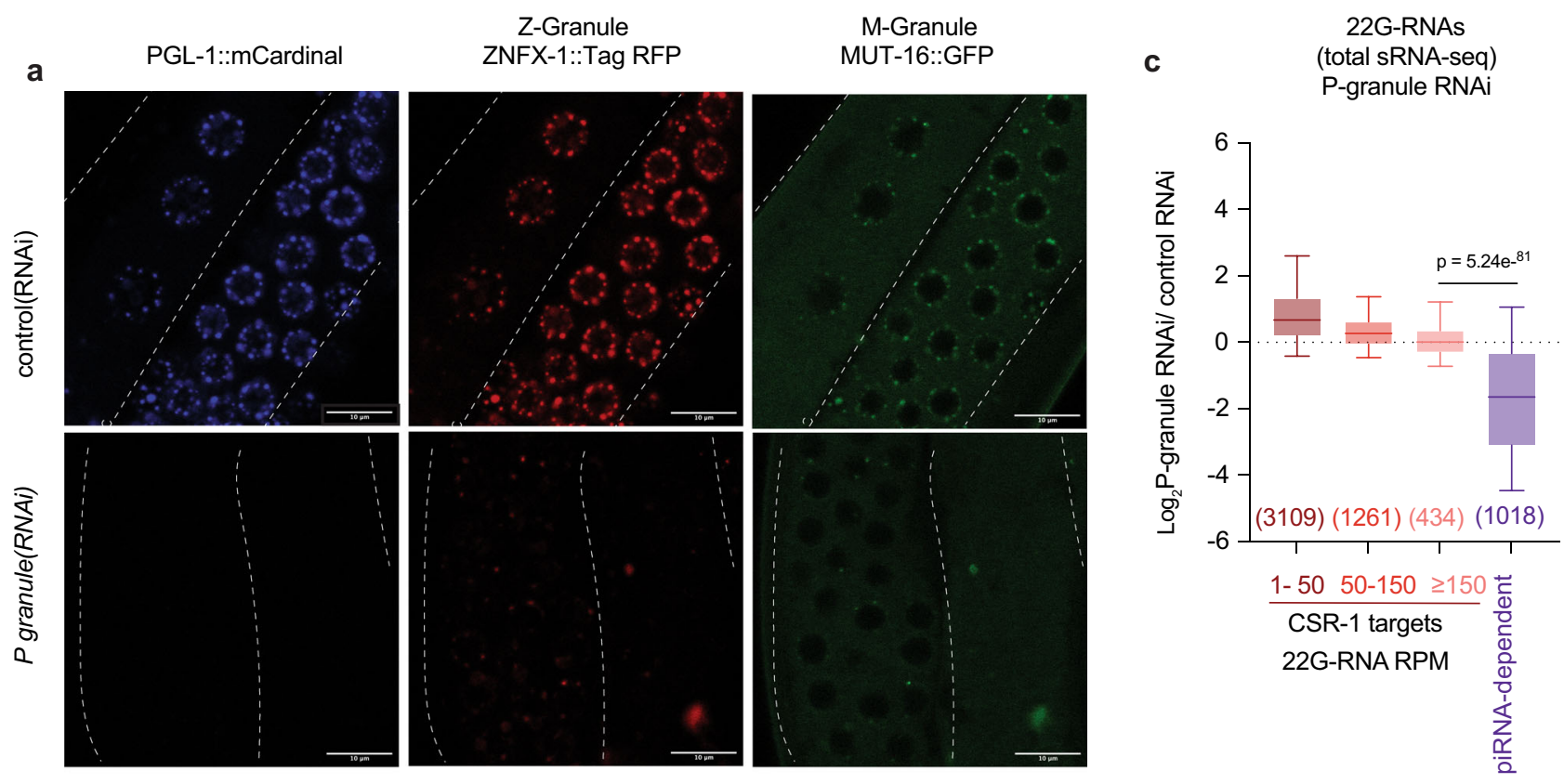

b

\section{P-Granule}

DEPS-1::GFP

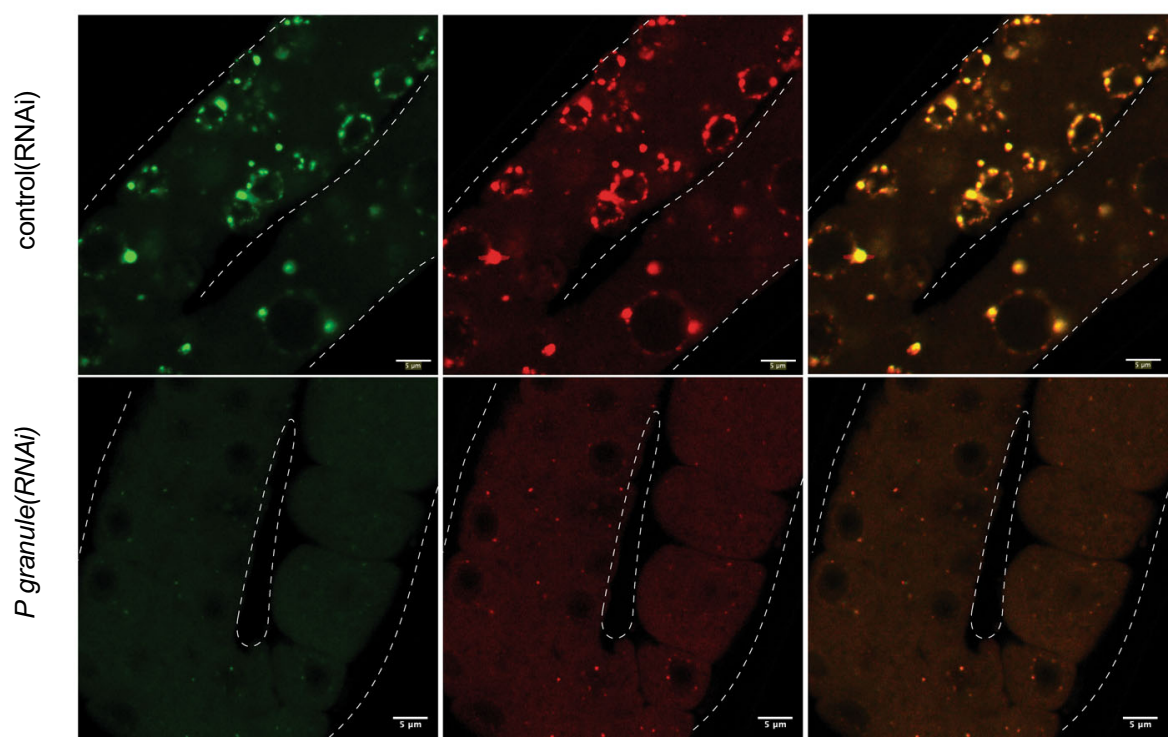

d
22G-RNAs

(RNA-seq, Campbell et.al. 2015) P-granule RNAi

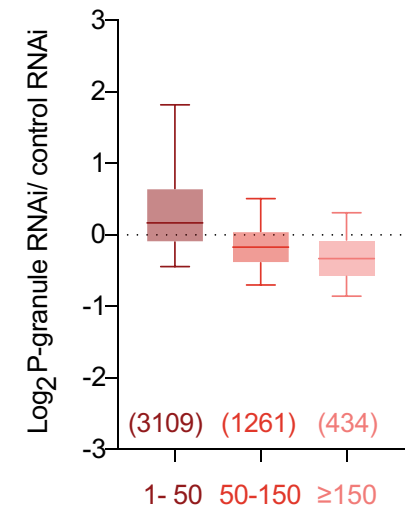

CSR-1 targets 22G-RNA RPM

Fig. 3 Biogenesis of CSR-1 22G-RNAs and the regulation of their targets do not require germ granule localization. a Live-fluorescent images of animals

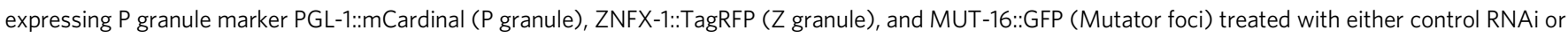
P granule RNAi ( $p g l-1, p g l-3, g / h-1$, and glh-4). All three granule types are depleted. Scale bars represent $10 \mu m$. $\mathbf{b}$ Live-fluorescent image of animals expressing P granule marker DEPS-1::GFP (P granule) and mCherry::CSR-1 treated with either control RNAi or P granule RNAi (pgl-1, pgl-3, glh-1, and glh-4). Scale bars represent $5 \mu \mathrm{m}$. CSR-1 is localized in both cytosol and P granule, and upon RNAi treatment, P granule localization of CSR-1 is lost while maintaining cytosolic localization. At least ten individual germlines were imaged for each condition in a, b. c Box plots showing the log 2 fold-change of total $22 \mathrm{G}-\mathrm{RNA}$ (sRNA-seq) upon P granule RNAi compared to control RNAi. The distribution for the 22G-RNA in CSR-1 IP for CSR-1 targets with 1-50 RPM, 50-150 RPM, or $\geq 150$ RPM (gene list in Supplementary Data 1) and piRNA-dependent 22G-RNA target genes ${ }^{66}$ are shown. Data is average of two biological replicates. $\mathbf{d}$ Box plots showing the $\log _{2}$ fold-change of mRNA expression (RNA-seq from Campbell et. al. ${ }^{41}$ ) upon P granule RNAi compared to control RNAi. The distribution for the 22G-RNA in CSR-1 IP for CSR-1 targets with 1-50 RPM, 50-150 RPM, or $\geq 150$ RPM. For both the box plots, the line indicates the median value, the box indicates the first and third quartiles, and the whiskers indicate the 5 th and 95th percentiles, excluding outliers. Two-tailed $P$-values were calculated using Mann-Whitney-Wilcoxon tests. The sample size $n$ (genes) is indicated in parentheses. Source data are provided as a Source Data file.

optimal and non-optimal codons using our experimental data from Late L4-staged worms. First, we calculated the normalized average relative synonymous codon usage (RSCU) for genes for different categories of high or low TE (Fig. 5b). Codons showing enrichment in genes with high TE $\left(\log _{2} \mathrm{TE} \geq 3\right)$ were considered optimal codons, and the ones under-represented were considered non-optimal codons (Fig. 5b). We confirmed that our classification of optimal/non-optimal codons correlated with tRNA copy 
a

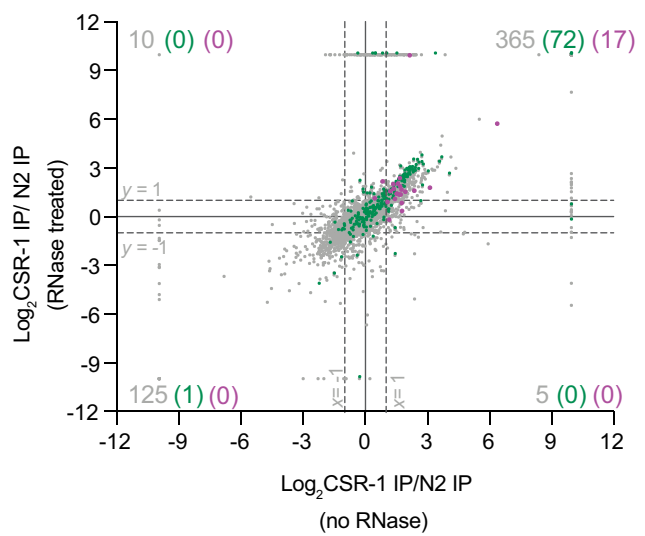

c
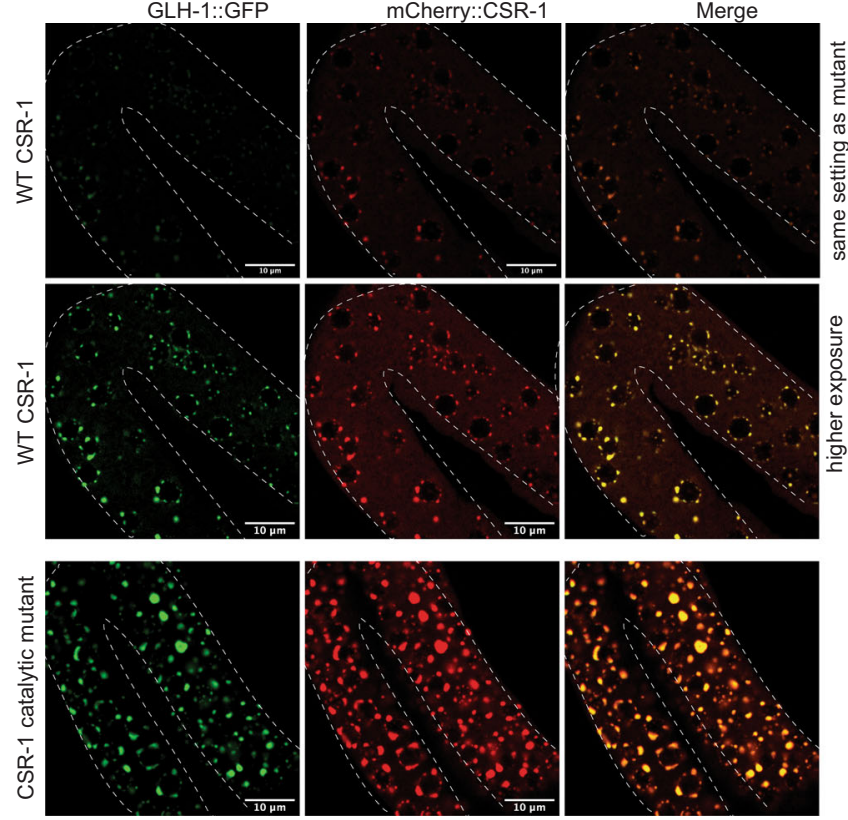

e

periodicity for the distance between 5'ends of 22G-RNAs and RPFs

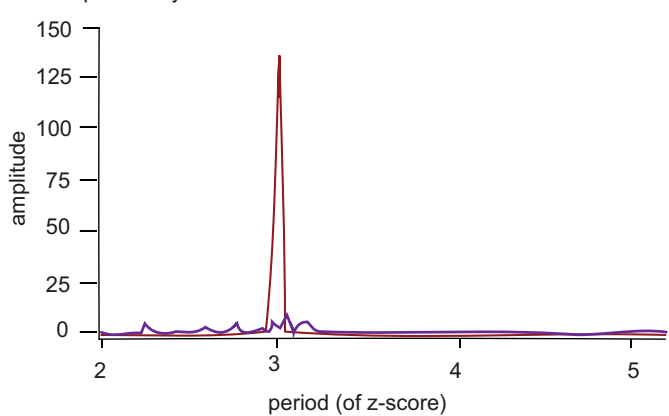

CSR-1 targets in CSR-1 IP

$P$ granule-dependent piRNA targets in HRDE-1 IP

number (Fig. 5c and Supplementary Fig. 8b, d) and tRNA expression in the late L4 worm population $(44 \mathrm{~h})$ as measured by GRO-seq (Fig. 5d and Supplementary Fig. 8c, e). We noticed that for codons with no tRNA cognates and requiring tRNA binding by wobble pairing, all optimal codons end with $\mathrm{C}$ and nonoptimal with U. Translation elongation is lower for those ending with a $\mathrm{U}^{47}$.
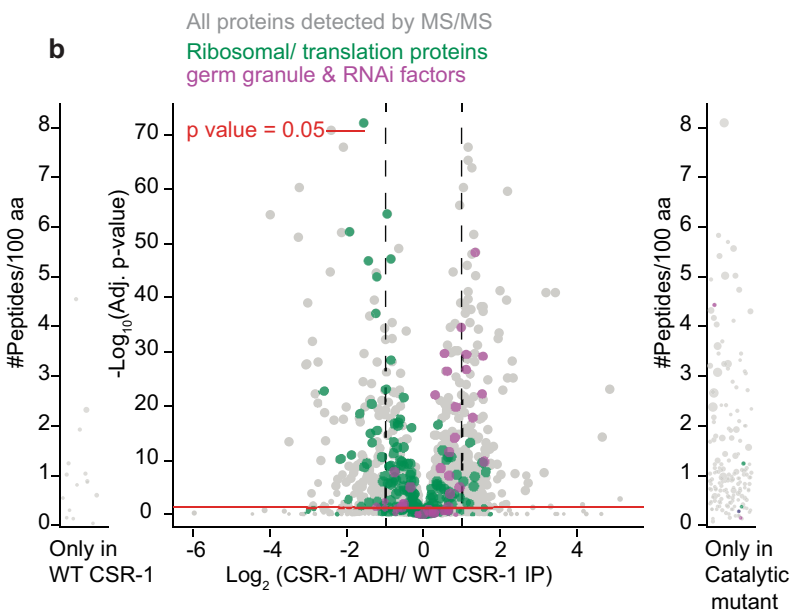

d

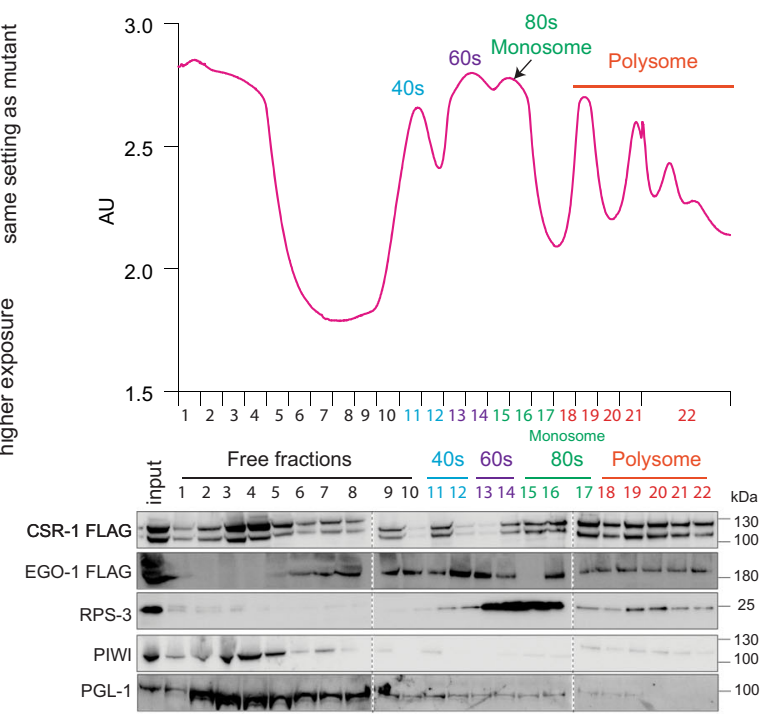

We then evaluated the codon usage of CSR-1 targets by comparing their normalized average RSCU to highly translated mRNAs. We found that non-optimal codons were enriched, and optimal codons were depleted in CSR-1 targets, suggesting that this might be an encoded feature of mRNA targets influencing the priming of 22G-RNA synthesis (Fig. 5e). Non-optimal codons are known to promote ribosome stalling ${ }^{48-50}$. To map differences in 


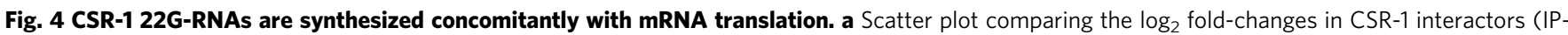
MS/MS) to control IPs performed in WT strain in the absence of RNase treatment ( $x$-axis) to the IPs performed after RNase treatment (Supplementary Data 2). Ribosomal proteins and translation regulators are highlighted in green, and germ granule proteins, including RNAi factors, are highlighted in magenta. Number in gray refers to all interactors with $\log _{2}$ fold-change of $\geq 1$ and $P$-value $\leq 0.05$ for each quadrant. The number in parenthesis is for ribosomal and translation-associated proteins enriched and granule and RNAi factors. $n=4$ biological replicates. $\mathbf{b}$ Volcano plot showing log 2 fold-change in enrichment values and corresponding significance levels for proteins co-purifying with CSR-1 ADH compared to WT CSR-1 (Supplementary Data 3). Ribosomal proteins and translation regulators are highlighted in green. Germ granule proteins, including RNAi factors, are highlighted in magenta. The size of the dots is proportional to the number of peptides used for the quantification. The linear model was used to compute the protein quantification ratio, and the red horizontal line indicates the two-tailed $P$-value $=0.05 . n=4$ biological replicates. $\mathbf{c}$ Live-fluorescent images showing localization and expression of GLH-1::GFP (P granule marker) and WT mCherry::CSR-1 or catalytic mutant mCherry::CSR-1 ADH. In WT, CSR-1 is localized to the cytosol and P granule. In CSR-1 catalytic mutant, CSR-1 is predominantly localized in enlarged P granules (Brightness of WT strain enhanced in middle panel for better visualization as the expression level of the mutant protein is higher than WT). At least five individual germlines were imaged for each strain. $\mathbf{d}$ Representative polysome profile indicating elution fractions with sub-monosomal, monosomal and polysomal complexes. Immunoblot for FLAG::CSR-1, FLAG::EGO-1 with anti-FLAG antibody and RPS-3, PIWI, and PGL-1 with their respective antibodies in sub-monosomal, monosomal, and polysomal fractions. The blots have been reproduced. e Periodogram based on Fourier transform for read-density around RPF 5' start position showing periodicity of CSR-1 22G-RNAs phasing with RPFs. P granule-dependent piRNA targets in HRDE-1 IP were used as control. Data is representative of two biological replicates. Source data are provided as a Source Data file.

22G-RNA biogenesis on sequences with optimal or non-optimal codons, we divided RPFs into two categories based on the presence of either an optimal or non-optimal codon at the A and $\mathrm{P}$ sites of the ribosome. We did not observe any specific bias at the last position of RPF (Supplementary Fig. 8f). We then mapped the distance between $5^{\prime}$ of 22G-RNAs and RPFs and observed a peak for the 5 '-end of 22G-RNAs downstream of RPF (29th position) when the $\mathrm{A}$ and $\mathrm{P}$ sites of the ribosomes are occupied by a non-optimal codon contrary to when optimal codons are present on A and $\mathrm{P}$ sites, which show no bias (Fig. $5 f$ ). This result suggests that the 22G-RNA production is preferentially initiated downstream of ribosomes especially occupying non-optimal codons that are difficult to translate, by CSR-1mediated slicing and recruitment of EGO-1.

Altogether, these observations suggest that translation and ribosome position dictate the production of CSR-1 22G-RNAs.

Increasing the translation efficiency of a CSR-1 target impairs CSR-1 22G-RNA biogenesis and function. To determine whether non-optimal codons directly affect TE and CSR-1 22G-RNA biogenesis, we altered the coding potential of a CSR-1 target. We examined $k l p-7$, which has the second-highest abundance of 22GRNAs loaded by CSR-1 and is post-transcriptionally regulated by CSR-1. KLP-7 is a kinesin-13 microtubule depolymerase and is required for spindle organization and chromosome segregation ${ }^{51}$. Overexpression of KLP-7 in the csr-1 mutant has been shown to cause microtubule assembly defects ${ }^{25}$. $k l p-7$ showed enrichment of non-optimal codons and depletion of optimal codons similarly to other CSR-1 targets (Supplementary Fig. 9a). We optimized the codon usage in $k l p-7$ by incorporating exclusively synonymous optimal codons (Supplementary Fig. 9a). We used CRISPR-Cas9 to replace endogenous $k l p-7$ isoform b with the modified $k l p-7$ codon-optimized ( $\left.k l p-7 \_c o\right)$ to avoid disrupting potential UTRmediated regulation.

To ascertain whether codon optimization of $k l p-7$ affected the TE, we performed RNA-seq and Ribo-seq from synchronized and sorted late L4 population $(44 \mathrm{~h})$. Indeed, we detected a twofold increase in the TE of $k l p-7$ mRNA in the $k l p-7$ co strain compared to WT (Fig. 6a). The TE of other CSR- -1 targets remained unaffected in the $k l p-7$ co strain, indicating that the effects observed are specific to $k \bar{l} p-7$ mRNA (Fig. $6 \mathrm{~b}$ ). In addition, KLP-7 protein levels were increased in two independent lines of $k l p-7$ _co compared to WT, consistent with increased translation (Supplementary Fig. 9b). We then measured the level of 22G-RNAs antisense to $k l p-7$ mRNA in the $k l p-7$ co strain compared to WT, and we observed a 1.4-fold decrease in 22G-RNAs (Fig. 6a). The levels of 22G-RNAs for other CSR-1 targets remained unaffected (Fig. 6b). Further, the significant decrease in 22G-RNAs on the optimized klp-7_co allele was observed in exons 3-6 and was accompanied by an increase in Ribo-seq peak height at those positions (Fig. 6c, d). The klp-7_co strain also showed increased $k l p-7$ mRNA level compared to WT (Fig. 6a), and we confirmed this result by quantitative reverse transcription PCR (RT-qPCR) (Supplementary Fig. 9c). These results suggest that CSR-1 targeting, and regulation are impaired on $k l p-7$ _co mRNA. To validate this, we performed $c s r-1$ RNAi and showed increased $k l p$ 7 mRNA levels in the WT strain but not in the klp-7_co strain (Fig. 6e), suggesting that CSR-1 slicer activity is reduced at $k l p-$ 7_co mRNA. The increased levels of $k l p-7$ mRNA correlated with a reduction in brood size (Supplementary Fig. 9d) and higher embryonic lethality at $25^{\circ} \mathrm{C}$ in $\mathrm{klp}-7$ _co strain compared to WT (Supplementary Fig. 9e), indicating the physiological relevance of $k l p-7$ mRNA targeting by CSR-1. Finally, to rule out any difference in the production of either 22G-RNAs or mRNA levels due to possible developmental defects between $k l p-7$ co and WT strain, we generated a heterozygote strain of $k l p-7$ _co with a fluorescent GFP marker on the balancer chromosome. We sorted heterozygote GFP-positive worms with one copy of modified $k l p-7$ _co and one copy of WT $k l p-7$ each and performed RNA-seq and sRNA-seq. We observed similar results with a 1.8 -fold increase in mRNA levels for $k l p-7$ co compared to the WT $k l p-7$ copy and a 1.25-fold decrease in 22G-RNA levels (Supplementary Fig. 9f, g). These results demonstrate that CSR-1 22G-RNA biogenesis and activity are reduced on mRNA templates with optimized codons and increased translation.

Altogether, these results suggest efficiently translating ribosomes block the access of CSR-1 to the mRNA template and thereby hamper 22G-RNA production and, in turn, affect gene regulation by CSR-1 during germline development.

\section{Discussion}

In this study, we have determined the rules governing germline mRNA targeting by CSR-1 and addressed the long-standing paradox of CSR-1 function as an anti-silencer or a slicer. We show a significant fraction of the slicing activity of CSR-1 is directed towards the production of 22G-RNAs antisense the coding sequence of its targets. We further dissected the mechanism of CSR-1 22G-RNA production. We demonstrated that the majority of the synthesis of 22G-RNAs occurs in the cytosol on translating mRNA templates with a low translation efficiency. CSR-1 slices the target mRNA occupied by ribosomes and initiates 22G-RNA biogenesis by priming RdRP EGO-1 
a

Translation efficiency

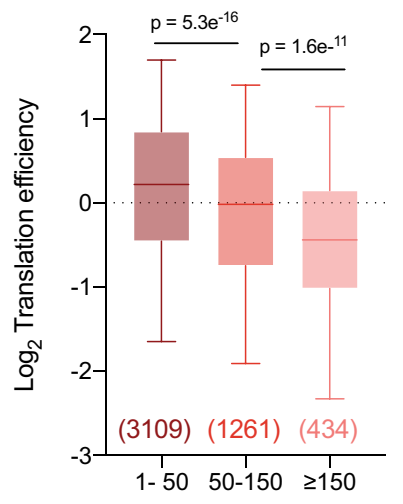

CSR-1 targets 22G-RNA RPM

C

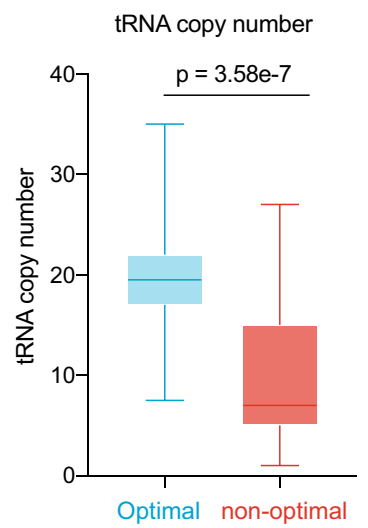

d

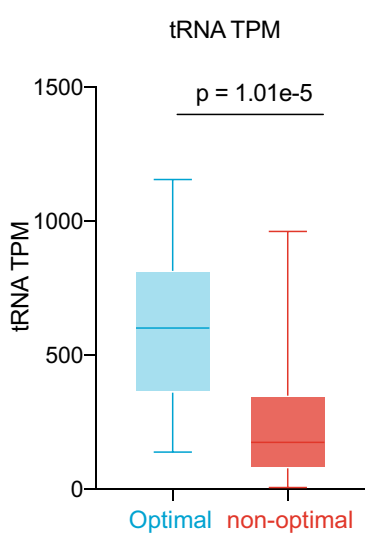

b

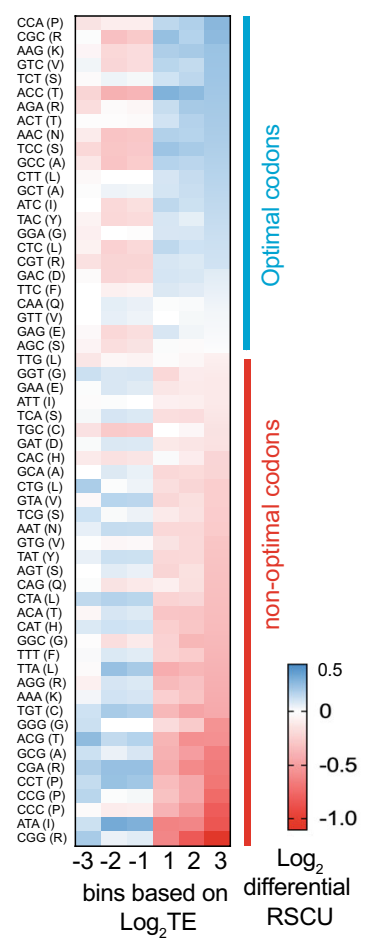

f

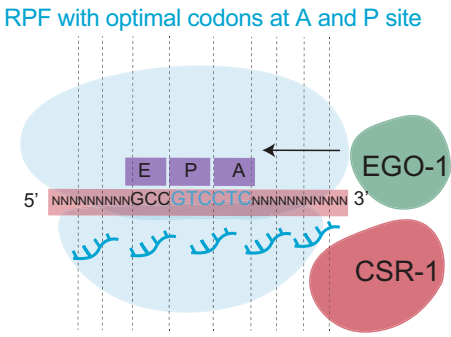

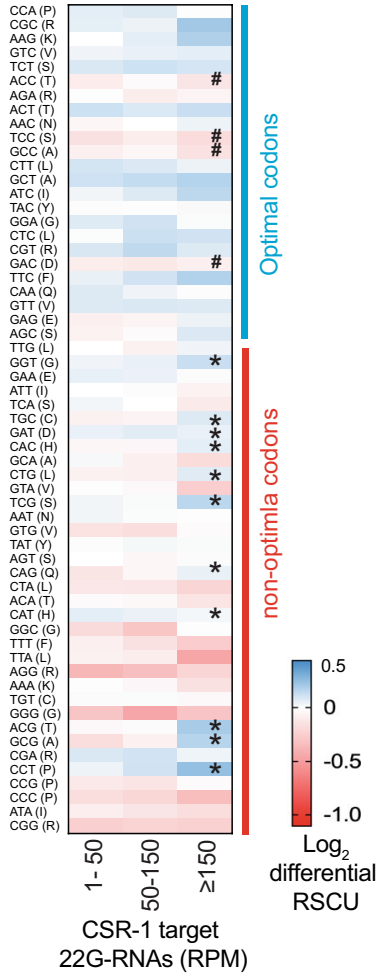

RPF with non-optimal codons at $\mathrm{A}$ and $\mathrm{P}$ site

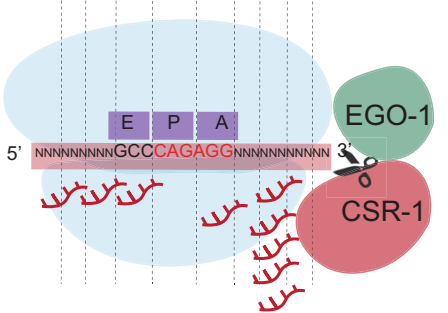

RPF with optimal codons at $A$ and $P$ site

RPF with non-optimal codons at $A$ and $P$ site

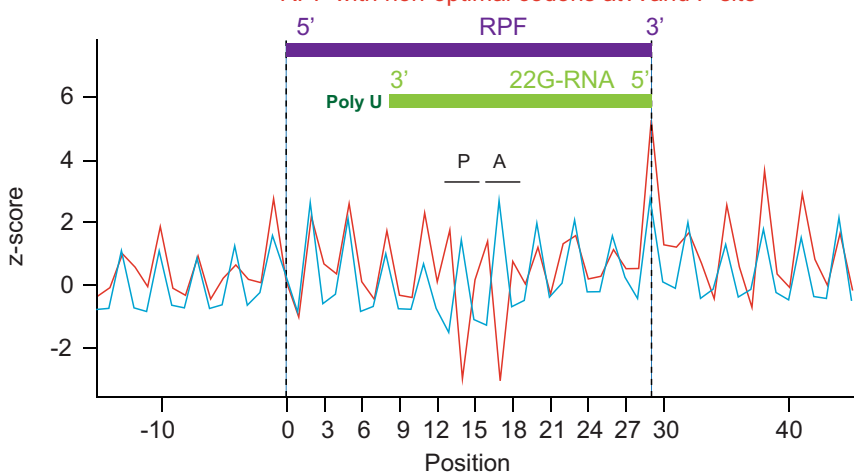

activity. Finally, we have determined how CSR-1 can preferentially target some germline mRNAs. We discovered that incorporating or avoiding non-optimal codons is a strategy adopted by germline mRNAs to be differentially regulated by CSR-1 22G-RNAs (Fig. 7). Overall, this study highlights the codon dependence and translational efficiency of mRNAs in the germline for the regulation of CSR-1 22G-RNAs biogenesis and, in turn, gene expression of the targets, which could have a significant bearing on germline gene regulation not just in worms but across species.
CSR-1 function as slicer and anti-silencer. We demonstrate that CSR-1 slicing activity regulates a fraction of targets, with a high abundance of 22G-RNA bound by CSR-1, post-transcriptionally in the germline, supporting the previous observation ${ }^{25}$. We further show that CSR-1 targets are enriched in CSR-1 direct interactors, including genes belonging to the CSR-1 pathway and CSR-1 itself. The upregulation of CSR-1 targets, therefore, may indirectly cause previously observed phenotypes, including chromatin defects ${ }^{14,25,52}$. Another recent study demonstrates that CSR-1 slicing activity is responsible for the decay of a larger 
Fig. 5 Highly translated mRNAs and optimal codons negatively correlate with CSR-1 22G-RNA abundance. a Translation efficiency Log 2 (RPF TPM/ mRNA TPM) for CSR-1 targets in WT strain. The distribution for the 22G-RNA in CSR-1 IP for CSR-1 targets with 1-50 RPM, 50-150 RPM, or $\geq 150$ RPM. The sample size $n$ (genes) is indicated in parentheses. The line indicates the median value, the box indicates the first and third quartiles, and the whiskers indicate the 5th and 95th percentiles, excluding outliers. Two-tailed $P$-values were calculated using Mann-Whitney-Wilcoxon tests. The sample size $n$ (genes) is indicated in parentheses. Data is an average of two biological replicates. b Heat map showing $\log _{2}$ fold-change in Relative Synonymous Codon Usage (RSCU) for all protein-coding genes categorized by increasing translational efficiency compared to genes showing neutral translational efficiency of 1 , as explained in methods. Codons are arranged in order of decreasing RSCU (top to bottom) in the category of $\log _{2}$ TE $\geq 3$. The blue line highlights optimal codons in genes with high TE, and the red line highlights non-optimal codons. c, d Box-plot showing the copy numbers for tRNAs for optimal or non-optimal codons (c), and the TPMs for tRNAs from the GRO-seq dataset for WT strain at the late 14 larval stage ( $44 \mathrm{~h}$ ) for optimal or non-optimal codons (d). The line indicates the median value, the box indicates the first and third quartiles, and the whiskers indicate the 5th and 95th percentiles, excluding outliers. Two-tailed $P$-values were calculated using Mann-Whitney-Wilcoxon tests. Two biological replicates. For codons with missing cognate tRNA, values have been adjusted by considering tRNA copy numbers and TPMs for tRNA recognizing these codons by wobble base pairing ${ }^{90}$. (see Supplementary Fig. $8 \mathrm{~b}, \mathrm{c}$ for non-adjusted values). e Heatmap similar to $\mathbf{b}$ showing $\log _{2}$ fold-change in relative synonymous codon usage for all CSR-1 targets (1-50, 50-150, and $\geq 150$ RPM of 22G-RNA) compared to genes showing neutral translational efficiency as explained in methods. "*" marks over-used non-optimal codons by CSR-1 targets and "\#" marks under-used optimal codons. f Plot showing the z-score for the read-density for the of 5' terminus of 22G-RNAs for CSR-1 targets relative to the start of 29-nt long Ribosomal protected fragments (RPF) with either optimal or non-optimal codons at their P and A site. The scheme shows possible initiation of 22G-RNA biogenesis after a slicing event downstream of RPF with non-optimal codons at the A and P sites. Source data are provided as a Source Data file.
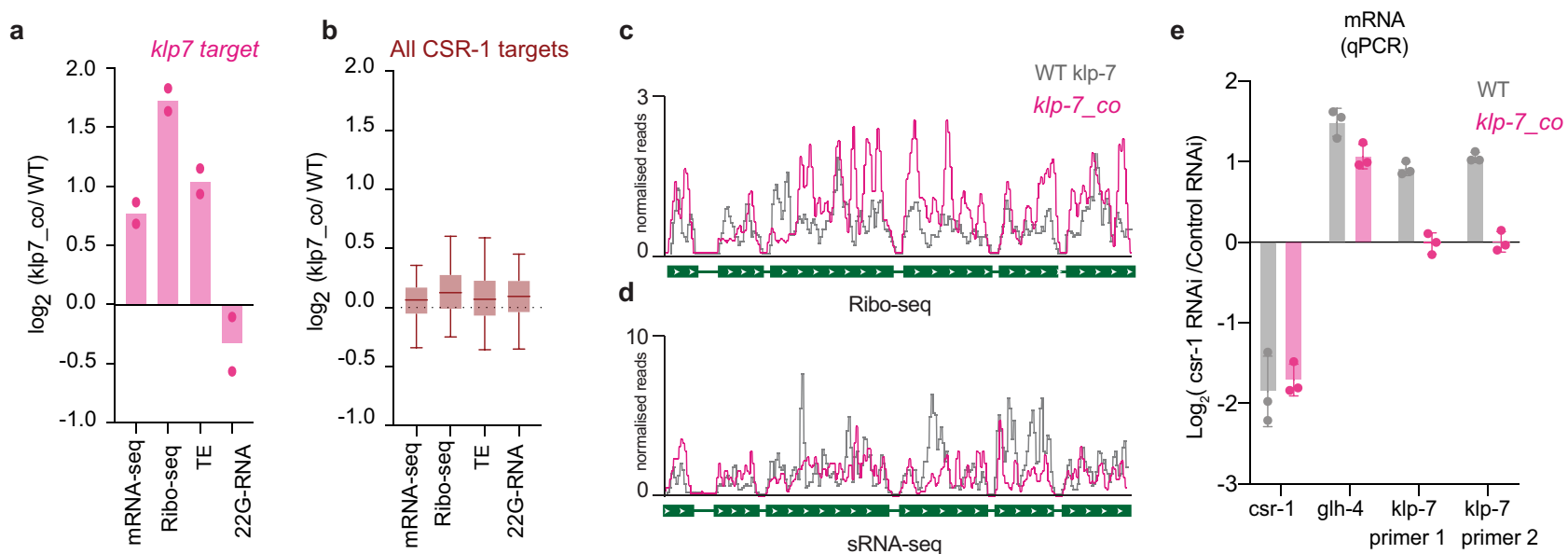

Fig. 6 Increasing optimal codon usage of CSR-1 target decreases small-RNA production. a, b Plot showing log fold-change for normalized reads for mRNAs from RNA-seq, RPF from Ribo-seq, and 22G-RNAs from sRNA-seq and differential Translational efficiency for klp-7 (top CSR-1 target) (a) and all CSR-1 targets (RPM $\geq 1, n=4803$ ) (b) for the strain with codon-optimized klp-7 (klp7_co) compared to WT strain. Two biological replicates are shown with their mean (a). For $\mathbf{b}$, the line indicates the median value, the box indicates the first and third quartiles, and the whiskers indicate the 5th and 95th percentiles, excluding outliers. Data is an average of 2 biological replicates. ( $n=4803$ CSR-1 targets except klp-7). c, d A genomic view of klp-7 showing Ribo-seq (c) and 22G-RNAs (d), normalized reads for the strain with codon-optimized klp-7 (klp7_co) in pink compared to WT strain in gray. Data is average of two biological replicates. e Log 2 fold-change in expression of csr-1, glh-4, and klp-7 upon csr-1 RNAi compared to control RNAi by qPCR in the WT strain and klp-7_co (strain with klp-7 codon optimization). Data are represented as mean \pm SD for three biological replicates. Source data are provided as a Source Data file.

number of maternally inherited CSR-1 target mRNAs in somatic blastomere in the embryos ${ }^{32}$. Our observations also suggest a catalytic-independent function of CSR-1 in preventing piRNAdependent chromatin silencing. Specifically, we showed that in the absence of CSR-1 protein, a different subset of CSR-1 target genes mostly comprised oogenic genes is misrouted into the piRNA pathway, which represses their expression at the transcriptional levels through the nuclear Argonaute HRDE-1. Therefore, in addition to the post-transcriptional regulation of germline mRNAs ${ }^{25}$, CSR-1 can also license the transcription of germline genes, which was hypothesized previously based on transgene analysis ${ }^{18,19}$ and shown directly here (Fig. 7). Mutation in the CSR-1 pathway was also shown to cause changes in the epigenetic landscape ${ }^{52,53}$. Given that HRDE-1 is known to promote the deposition of histone modifications associated with gene silencing, the effects observed upon mutation in components of the CSR-1 pathway might be the results of CSR-1 anti-silencing function. The majority of CSR-1 protected genes include oogenic genes, which initiate their transcription during the developmental stage analyzed in this study. We predict that more oogenic genes might be protected by CSR-1 at a later time point but is difficult to study due to developmental defects accumulated at these later timepoints ${ }^{25}$. The CSR-1 protected targets do not overlap significantly with CSR-1 sliced targets. We, therefore, propose that CSR-1 slicer and anti-silencer function co-exist to regulate different germline gene expression programs. This regulation is also potentially spatially compartmentalized due to different functions of CSR-1 in P granules and cytosol, as discussed in another paragraph below. Further studies are required to uncouple the impact of the slicer and anti-silencer function using tools depleting CSR-1 spatially and temporally. CSR-1 is not the only mechanism that might license germline mRNAs. Indeed, apart from CSR-1 targeting of mRNAs ${ }^{16}$, other mechanisms have been proposed to protect germline mRNA from piRNA silencing, including PATCs sequences in introns ${ }^{17}$, and not yet completely defined features in coding sequence ${ }^{15}$. Therefore, that might also 


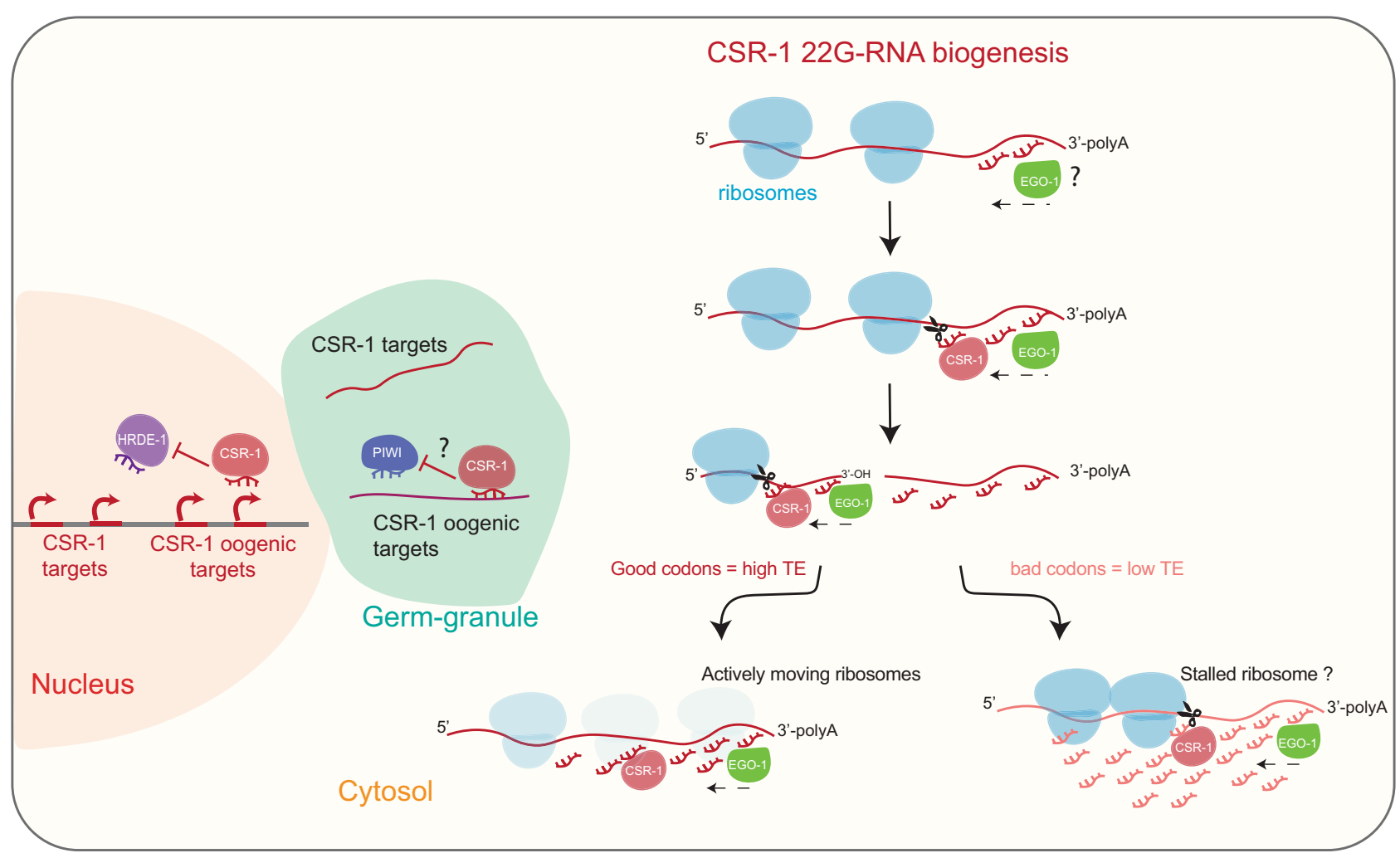

Fig. 7 Model illustrating biogenesis of CSR-1-22G-RNA in the cytosol. CSR-1 targets most of the germline-expressed genes. CSR-1 22G-RNAs are produced from mRNAs engaged in translation in the cytosol. We propose EGO-1 initiate 22G-RNA biogenesis at the 3UTR on every actively translating mRNAs or by being recruited on specific 3UTR sequences by yet unknown mechanism. However, to produce 22G-RNAs on coding sequence, CSR-1 slicing activity is required on the mRNA template. Codon usage and translation efficiency antagonistically regulate levels of 22G-RNAs production on different CSR-1 targets. We propose that CSR-1 can interact with ribosomes and the slicing event is more biased downstream of a possible stalled ribosome occupying a non-optimal codon site. CSR-1 slicer activity can regulate gene expression of few top targets, which further depends on the 22G-RNA levels. Additionally, CSR-1 can protect a set of mainly oogenic genes from piRNA-mediated HRDE-1 transcriptional silencing in a catalytic-independent manner.

explain why the removal of CSR-1 does not affect a large number of mRNAs.

Biogenesis of CSR-1 22G-RNAs. In this study, we have now established that the majority of Argonaute CSR-1 slicing activity cleaves target mRNAs to trigger the generation of RdRPdependent 22G-RNAs on the gene body. We propose that CSR1 slicer activity is required to generate new $3^{\prime}-\mathrm{OH}$ ends along the gene transcript to facilitate the initiation of 22G-RNA synthesis by RdRP (EGO-1) towards the 5'-end of the mRNA target. This is consistent with previous in vitro RdRP analysis showing that non-polyadenylated 3'-OH ends of RNAs served as better substrates for $22 \mathrm{G}-\mathrm{RNA}$ synthesis ${ }^{24}$, suggesting that the cleavage of RNA may be vital for the processivity of RdRPs. Based on these results, we speculate that no primary small RNAs are required to generate CSR-1 22G-RNAs along the mRNA sequence. Instead, CSR-1 catalytic activity triggers the synthesis of $22 \mathrm{G}-\mathrm{RNAs}$ by the RdRP EGO-1, starting from the 3'UTR of the target transcripts. Even if the catalytic activity of CSR-1 is required to generate 22GRNAs along the gene body of target transcripts, it is still unknown what triggers the recruitment of EGO-1 on the 3'UTR. Primary small RNAs, which are yet to be identified, might prime the activity of EGO-1. Alternatively, EGO-1 might produce low levels of $22 \mathrm{G}$-RNAs from the polyadenylated tail of mRNAs instead of cleaved 3'OH end products. Thus, these low levels of 22G-RNAs, which are then loaded into CSR-1, can initiate the production of 22G-RNAs along the gene body. RNA binding proteins and/or other unknown factors together with specific sequences in the
3UTR might also recruit and initiate EGO-1-dependent 22GRNAs from the 3'UTR of selected mRNAs.

The role of translation and codon usage in CSR-1 22G-RNA biogenesis. Germ granules are thought to be the site for all 22GRNA synthesis and have been shown to be essential for the synthesis of piRNA-dependent 22G-RNAs. Whether CSR-1 22GRNAs are also generated in germ granules is still unknown. In our current study, we show that CSR-1 22G-RNAs are synthesized in phase with ribosomes on actively translating mRNAs. $\mathrm{P}$ granules are known to be depleted of translating mRNAs, and $\mathrm{P}$ granule enriched mRNAs become translationally active upon $\mathrm{P}$ granule exit $^{42}$. In addition, biochemical and proteomic characterizations of other cytoplasmic granules such as $\mathrm{P}$ bodies also show that those granules are depleted of ribosomal proteins ${ }^{54}$. We further showed that both CSR-1 and RdRP, EGO-1, are present in the polysome fractions, indicative of their interaction with translating mRNAs. We also observed a characteristic three-nucleotide periodicity between the start position of CSR-1-associated 22GRNAs and RPFs, typical of the ribosomal footprint. A similar three-nucleotide periodicity has been observed for other cotranslational events like $5^{\prime}$ to $3^{\prime}$ exonucleolytic decay of decapped mRNAs ${ }^{55}$.

In contrast to CSR-1, we found PIWI was not enriched in polysome fractions, and downstream argonaute HRDE-1-bound piRNA-dependent 22G-RNAs are randomly distributed with respect to 5 'end of the RPF, indicating that the results obtained with CSR-1 22G-RNAs are not due to sequence bias. We also show that RNAi of $\mathrm{P}$ granule components, which disrupt germ 
granules and CSR-1 granule localization, results in the impairment of piRNA-dependent 22G-RNAs but not CSR-1-associated 22G-RNAs. Taken together, these results allowed us to conclude that the majority of CSR-1 22G-RNA biogenesis occurs in the cytosol co-translationally.

The co-translational synthesis of CSR-1 22G-RNAs raises the question of how CSR-1-22G-RNA biogenesis machinery is able to cope with the presence of ribosomes on the target transcripts. We show that non-optimal codons in germline mRNAs enhance the capacity of CSR-1 to prime the synthesis of EGO-1-dependent 22G-RNAs along the gene body. In fact, the translation efficiency of CSR-1 targets inversely correlates with 22G-RNA levels. We propose that the use of non-optimal codons by CSR-1 targets and priming of 22G-RNAs at stalled positions is a way to cope with the ribosomal presence on the target transcripts. Therefore, sequences that promote ribosome stalling promote targeting by CSR-1 to recruit EGO-1 on coding sequence to synthesize $22 \mathrm{G}$ RNAs. To test this hypothesis, we have shown that the substitution of non-optimal codons with optimal codons is sufficient to allow germline mRNAs to escape CSR-1-dependent regulation. However, it is still unclear how EGO-1 initiates the synthesis of 22G-RNAs at the 3'-end of RPF, and this requires further investigation. One possibility is that CSR-1 and EGO-1 might coordinate their activity with the Ski complex, which extracts mRNA from $80 \mathrm{~S}$ ribosomal complexes in a $3^{\prime} \rightarrow 5^{\prime}$ direction facilitating exosomal degradation ${ }^{56}$. Additionally, ribosome-phased endonucleolytic cuts possibly produced by the ribosome by the process called ribothrypsis, at the exit site of the mRNA ribosome channel may facilitate EGO-1 movement on transcript occupied by stalled ribosome ${ }^{57}$. There is increasing evidence that the translation machinery associates with the Argonautes and small-RNA biogenesis factors. Ribosome movement on translating mRNAs resolves mRNA structure to provide accessibility to Argonaute AGO2 downstream of the ribosome and promote AGO2-target interaction ${ }^{58,59}$. Another report showed that RNAi can occur co-translationally with an accumulation of ribosomes upstream of the dsRNA targeted region ${ }^{60}$. Ribosomes have been shown to coordinate with piRNA biogenesis factors in mouse testes to achieve endonucleolytic cleavage of non-repetitive long RNAs to produce pachytene piRNAs $^{61}$. In plants, 22-nt siRNAs can repress translation, leading to induction of transitive small-RNA amplification by RNA-dependent RNA polymerase 6 (RDR6) ${ }^{62}$. Another recent report in plants showed that microRNA targeting recruits a double-strand RNA binding protein, which induces ribosome stalling, and the ribosome stalling enhances the generation of secondary small RNAs ${ }^{63}$. Therefore, we propose that the regulation of small-RNA biogenesis by ribosome occupancy and codon usage of the target transcript might be a general strategy adopted across evolution.

Granule vs. cytosolic functions of CSR-1. We found that the slicer activity of CSR-1 and 22G-RNA biogenesis at germline mRNA targets are independent of $\mathrm{P}$ granules. This raises the question on the function of CSR-1 in germline granules. CSR-1 might be enriched in $\mathrm{P}$ granules of adult gonads to prevent CSR-1 slicer activity on the majority of germline mRNAs. Indeed, only $7.7 \%$ of CSR-1-dependent 22G-RNA targets are significantly regulated by CSR-1 slicer activity in adults. Moreover, the majority of these targets are CSR-1-interacting proteins, suggesting a negative feedback regulation of the CSR-1 pathway. This is in contrast with the recently described function of the maternally delivered CSR-1 in the embryo, which exclusively localizes in the cytosol of the somatic blastomere, where it cleaves and clears hundreds of maternal mRNA targets ${ }^{32}$. Therefore, we propose that CSR-1 slicer activity on mRNA targets is partially suppressed in the germline by titrating away a part of CSR-1 in P granules and primarily serves to generate interacting small RNAs in the cytosol that fully operates intra-generationally in the embryo. This also explains why despite targeting almost all germline genes, CSR-1 catalytic activity regulates the expression of only a few in the germline. In addition, CSR-1 localization in the $\mathrm{P}$ granule might serve to antagonize piRNA-dependent targeting on germline mRNAs and therefore license those transcripts to be translated in the cytosol. Indeed, we have shown that most of the piRNA-dependent 22G-RNAs are generated in P granules, and we propose that the competition between CSR-1 and PIWI might occur in $\mathrm{P}$ granules.

\section{Methods}

C. elegans strains and maintenance. Strains were grown at $20^{\circ} \mathrm{C}$ on NGM plates seeded with $E$. coli OP50 using standard methods ${ }^{64}$ unless otherwise stated. The wild-type reference strain used was Bristol N2. A complete list of strains used in this study is provided in Supplementary Data 5.

Generation of CRISPR-Cas9 lines. Cas9-guide RNA (gRNA) ribonucleoprotein complexes were microinjected into the hermaphrodite syncytial gonad as described previously ${ }^{65}$, and gRNA design and in vitro synthesis were done following the protocol detailed in ${ }^{66}$. For the introduction of a $c s r-1(D 769 A)$ mutation ${ }^{66}$ in $3 \times f l a g:: h a:: c s r-1$ animals, we used a single-stranded oligonucleotide repair template ordered from IDT as standard $4 \mathrm{nM}$ ultramer oligo. For the endogenous $k l p-7$ gene replacement, we used two gRNAs, each one respectively targeting a region at the 5 and $3^{\prime}$ of the $k l p-7$ isoform b gene. A PCR repair template containing $33 \mathrm{bp}$ homology arms was directly amplified from a plasmid containing a codonoptimized version of $k l p-7$ ( $k l p-7 \_c o$ synthetic gene) synthesized from GenScript (Supplementary Data 6).

Mix concentrations were adapted from ${ }^{67}$. In brief, $10 \mu \mathrm{L}$ mixes typically contained the following final concentrations: $0.1 \mu \mathrm{g} / \mu \mathrm{L}$ Cas9-NLS protein (TrueCut $\mathrm{V} 2$, Invitrogen), $100 \mathrm{ng} / \mu \mathrm{L}$ in vitro transcribed target-gene gRNA, $80 \mathrm{ng} / \mu \mathrm{L}$ of target-gene ssODN repair template or $300 \mathrm{ng} / \mu \mathrm{L}$ target-gene double-stranded DNA repair template and $80 \mathrm{ng} / \mu \mathrm{L}$ pRF4 (roller marker). Cas 9 and the target-gene gRNA were pre-incubated $10-15 \mathrm{~min}$ at $37^{\circ} \mathrm{C}$ before the addition of the other components to the mixture. dsDNA repair templates were subjected to a melting/ annealing step ${ }^{67}$ before addition to the final mix. A detailed list of gRNAs, singlestranded DNA, and double-stranded DNA repair templates and primers used for genotyping are provided in Supplementary Data 6.

RNAi. RNAi clones for ego-1 and csr-1 used in this study were obtained from the Ahringer library ${ }^{68}$. For quadruple P granule RNAi ( $p g l-1$, $p g l-3$, glh-1, and $g l h-4$ ), pDU49 clone (gift from Updike lab ${ }^{40}$ ) was used. An empty vector (L4440) was used as a control in all of our RNAi experiments. RNAi experiments were performed by growing a synchronous population of L1 larvae on Petri dishes with NGM and IPTG $(15 \mathrm{~cm})$ seeded with concentrated RNAi food. For $c s r-1$ and ego- $1 \mathrm{RNAi}$, worms were grown from L1 to late L4 stage on RNAi food at $20^{\circ} \mathrm{C}$. For P granule RNAi, worms were grown for two generations at $25^{\circ} \mathrm{C}^{40}$. Post-RNAi treatment, worms were harvested and sorted on COPAS biosorter to enrich late L4 larvae. RNAi efficacy was confirmed by RT-qPCR.

ego-1 RNAi and auxin-induced CSR-1 degradation. For ego-1 RNAi worms were grown from $\mathrm{L} 1$ to $38 \mathrm{~h}$ post-hatching on RNAi or control food on IPTG containing plates and then washed twice with M9 buffer and then shifted to either Auxin plates or Ethanol plates (containing $500 \mu \mathrm{M}$ auxin, $0.5 \%$ Ethanol or only $0.5 \%$ Ethanol respectively) to deplete degron-tagged CSR-1 by auxin-induced degradation as described before ${ }^{32}$. Plates were seeded with respective ego- 1 RNAi or control RNAi food. Auxin-induced degradation was performed for $6 \mathrm{~h}$. Worms were then harvested, washed with M9 buffer, and sorted on COPAS biosorter to enrich for Late L4 larval population. CSR-1 depletion was confirmed by live imaging.

CSR-1 expression recovery post-auxin-induced degradation. A synchronous population of degron-tagged CSR-1 strain was grown on NGM plates containing $500 \mu \mathrm{M}$ auxin, $0.5 \%$ ethanol from $\mathrm{L} 1$ to $38 \mathrm{~h}$ post-hatching to degrade degrontagged CSR-1. After $38 \mathrm{~h}$, worms were washed thrice with M9 buffer and divided into three parts. $1 / 3^{\text {rd }}$ worms were immediately sorted on COPAS biosorter to enrich for a synchronous population for $0 \mathrm{~h}$ recovery time point of CSR-1 expression. The rest of the worms were seeded on two NGM plates and allowed to grow in the absence of auxin induction for 5 or $10 \mathrm{~h}$ to recover CSR-1 expression. Worms were washed with M9 buffer at respective time points and sorted using COPAS biosorter to enrich for a synchronized population for each time point. CSR-1 expression was monitored using live imaging. 
Brood-size assay. For the brood size, single L1 larvae were manually picked and placed onto NGM plates seeded with E. coli OP50 and grown at $20^{\circ} \mathrm{C}$ or $25^{\circ} \mathrm{C}$ until adulthood and then transferred on a new plate every $24 \mathrm{~h}$ for a total of 2 transfers. The brood size of each worm was calculated by counting the number of embryos and larvae laid on the three plates. Embryonic lethality was measured by counting the number of the unhatched embryo (dead) $24 \mathrm{~h}$ post laying compared to total embryos laid.

Counting of oocytes in population. For the WT (N2) and CSR-1 catalytic mutant, germlines of adult worms ( $72 \mathrm{~h}$ post-hatching) were dissected and stained with DAPI, and the number of oocytes was counted.

Sorting. Large populations of the Late L4 larvae stage from the synchronized population were sorted using the COPAS BIOSORT instrument (Union Biometrica), according to the manufacturer's guidelines. The population was sorted using two size parameters, Time of flight (TOF) and extinction. The stage of the sorted population was validated by counting worms under a microscope by scoring features like closed vulva and absence of oocytes as a characteristic of late L4 stage larvae. First-generation homozygotes for CSR-1 KO or CSR-1 ADH were sorted by excluding GFP-positive heterozygote worms. klp-7_co heterozygote strain was sorted using GFP marker, and GFP-positive worms were sorted.

Imaging. Transgenic worms were mounted on 2\% agarose pads in a drop of M9 with $1 \mathrm{mM}$ Levamisole. Images were acquired on ZEISS LSM 700 microscope with a $\times 40$ objective or $\times 63$ objective for the PGL-1::mCardinal; ZNFX-1:: TagRFP; MUT-16::GFP, DEPS-1::GFP; mCherry::CSR-1, GLH-1::GFP;mCherry::CSR-1 and GLH-1::GFP; mCherry::CSR-1 ADH. Images were acquired using the ZEISS ZEN software and processed using ImageJ v.2.0.0. mCherry::CSR-1 in mut-16 and $z n f x-1$ mutant background were imaged on Zeiss Axio Imager M2 and were acquired using MetaMorph software. All strains are listed in Supplementary Data 5. For counting oocytes, dissected gonads were mounted in DAPI containing Vectashield mounting medium, and oocytes were counted by visualizing on Zeiss Axio Imager M2.

Western blotting. Worms were lysed in 1x NuPAGE LDS sample buffer (Ther moFisher Scientific) and heated at $90^{\circ} \mathrm{C}$ for $10 \mathrm{~min}$. Any debris was removed by centrifuging at $18,000 \times g$. $\sim 50 \mu \mathrm{g}$ of protein extracts was then resolved on precast NuPAGE Novex 4-12\% Bis-Tris gels (Invitrogen, NP0321BOX). The proteins were transferred to a nylon membrane with the semidry transfer Pierce Power System (ThermoFisher Scientific) using the pre-programmed method for high-molecularmass protein. The primary antibodies used included anti-KLP- $7^{25}$ (1:1000 dilution) (a gift from the Desai laboratory), anti-tubulin (Ab6160, Abcam) (1:1000 dilution), anti-GAPDH (Ab125247, Abcam) (1:2000 dilution), anti-PGL-1 ${ }^{69}$ (1:2000 dilution) (a gift from the Strome laboratory), anti-PRG-170 (1:2000 dilution) (a gift from the Mello laboratory), anti-Flag (F3165, Sigma) (1:1000 dilution), anti-RPS-3 (ab128995, Abcam) (1:3000 dilution) and the secondary antibodies used included anti-rabbit (31460, Pierce) (1:10000 dilution), anti-mouse (31430, Pierce) (1:10000 dilution) and anti-rat (A9037, Sigma) (1:10000 dilution) HPR antibodies. The SuperSignal West Pico PLUS Chemiluminescent Substrate was used to detect the signal using a ChemiDoc MP imaging system (Biorad).

RNA extraction. For total RNA extraction, synchronous and sorted populations of $\sim 1000$ worms as described for individual experiments were frozen in dry ice with TRIzol $^{\mathrm{TM}}$ (Invitrogen, Ref. 15596026). After five repetitions of freeze and thaw, total RNA was isolated according to the manufacturer's instructions. For RNA extraction after IP, TRI Reagent was directly added to beads, and RNA extraction was performed as per the manufacturer's instructions. For RNA used for RNA-seq or RT-qPCR, DNase treatment was performed using a maximum of $10 \mu \mathrm{g}$ RNA treated with $2 \mathrm{U}$ Turbo DNase (Ambion) at $37^{\circ} \mathrm{C}$ for $30 \mathrm{~min}$ followed by acid phenol extraction and ethanol precipitation. An Agilent 2200 TapeStation System was used to evaluate the RIN indexes of all of the RNA preps, and only samples with RNA integrity numbers (RIN) $>8$ were used for downstream applications.

Quantitative reverse transcription PCR (RT-qPCR). Reverse transcription was performed according to manufacturer's instructions using M-MLV reverse transcriptase (Invitrogen, Ref. 28025013), and qPCR was performed using Applied Biosystems Power up SYBR Green PCR Master mix following the manufacturer's instructions and using an Applied Biosystems QuantStudio 3 Real-Time PCR System and analyzed using QuantStudioTM Design and Analysis software V 2.2. Primers used for qPCR are listed in Supplementary Data 7.

IP/total- sRNA-seq. Total RNA from at least 1000 sorted worms with RIN $>9$ was used to generate small-RNA libraries. For 22G-RNAs from IP, IP was performed using $\sim 10,000$ synchronized and sorted worms for FLAG-CSR-1 or $\sim 70,000$ for GFP-HRDE-1. Worms were lysed in small-RNA IP buffer (50 mM HEPES pH 7.5, $500 \mathrm{mM} \mathrm{NaCl}, 5 \mathrm{mM} \mathrm{MgCl}$, $1 \%$ NP-40, 10\% glycerol, 1x Halt protease inhibitors and RNaseIn $40 \mathrm{U} / \mathrm{mL}$ ), using a chilled metal dounce. Crude lysates were cleared of debris by centrifuging at $18,000 \times g$ at $4^{\circ} \mathrm{C}$ for $10 \mathrm{~min}$. Ten percent of the extract was saved as input, and total RNA was extracted using TRIzol ${ }^{\mathrm{TM}}$ as described above. The rest of the extract was incubated with $30 \mu \mathrm{l}$ of Anti-FLAG M2 Magnetic Agarose Beads suspension (Sigma M8823) or $25 \mu \mathrm{l}$ GFP-Trap Magnetic Agarose (Chromotek gtma-10) for FLAG-CSR-1 or GFP-HRDE-1 respectively, for $1 \mathrm{~h}$ at $4^{\circ}$ C. After four washes of the beads with the small-RNA IP buffer, the RNA bound to the bait was extracted by adding TRIzol ${ }^{\mathrm{TM}}$ to beads as described above. The library preparation was performed essentially as described previously ${ }^{66}$. Amplified libraries were multiplexed to purify further using PippinPrep DNA size selection with $3 \%$ gel cassettes and the following parameters for the selection: BP start (115); $\mathrm{BP}$ end (165). The purified libraries were quantified using the Qubit Fluorometer High Sensitivity dsDNA assay kit (Thermo Fisher Scientific, Q32851) and sequenced on a NextSeq-500 Illumina platform using the NextSeq 500/550 High Output v2 kit 75 cycles (FC-404-2005).

IP and radiolabeling of sRNA. IP was performed as described above for FLAG: CSR-1 and FLAG:::CSR-1 ADH. Ten percent of total extract and IP was processed for western blotting. The rest of the IP was used for extracting RNA using TRIzol as described above. RNA was treated with polyphosphatase to generate monophosphate $5^{\prime} \mathrm{ends}^{66}$. 5'end of RNA from the above step was labeled using $\gamma \mathrm{P}^{32}$-ATP using T4-polynucleotide kinase (EK0031, Thermofisher Scientific) as per manufacturer's instructions. Labeled RNA was purified using $1.8 \mathrm{x}$ SPRI beads with isopropanol and resuspended in $10 \mu \mathrm{L}$ water. TBE Urea loading buffer (Thermofisher Scientific) was added to the sample, and RNA denatured at $70^{\circ} \mathrm{C}$ for $5 \mathrm{~min}$ and then resolved on Novex ${ }^{\mathrm{TM}} 15 \%$ TBE Urea gel (Thermofisher Scientific). The resolved gel was exposed on a Phosphor screen and scanned on Typhoon FLA 9000 scanner.

Gro-seq. One thousand synchronized and sorted Late L4 worms for WT (N2), csr1 catalytic mutant and $c s r-1$ KO were collected as described above. Nuclear Run-on reaction was performed by incorporating $1 \mathrm{mM}$ Bio-11-UTP, followed by RNA extraction and biotinylated nascent RNA enrichment as described previously ${ }^{32}$ Libraries were prepared by repairing 5 '-OH of fragmented RNAs by Polynucleotide Kinase (Thermo scientific), followed by $3^{\prime}$ and $5^{\prime}$ adapter ligation as described previously ${ }^{32}$. Adapter ligated RNA was reverse transcribed using SuperScript IV Reverse Transcriptase (Thermo Fisher Scientific) following manufacturer conditions, except that reaction was incubated for $1 \mathrm{~h}$ at $50^{\circ} \mathrm{C}$. cDNA was PCR amplified with specific primers using Phusion High fidelity PCR master mix 2x (New England Biolab) for 18-20 cycles. Libraries were analyzed on Agilent 2200 TapeStation System using high-sensitivity D1000 screentapes and quantified using the Qubit Fluorometer High Sensitivity dsDNA assay kit (Thermo Fisher Scientific, Q32851). Multiplexed libraries were sequenced on a NextSeq-500 Illumina platform using the NextSeq 500/550 High Output v2 kit 75 cycles (FC-404-2005).

Strand-specific RNA-seq library preparation. DNase-treated total RNA with RIN $>8$ was used to prepare strand-specific RNA libraries. Ribosomal and mitochondrial rRNAs were depleted using a custom RNAse-H-based method to degrade rRNAs using complementary oligos as described previously ${ }^{66}$.

Strand-specific RNA libraries were prepared using at least $100 \mathrm{ng}$ of rRNA depleted RNAs using NEBNext Ultra II Directional RNA Library Prep Kit for Illumina (E7760S). RNA libraries were analyzed on Agilent 2200 TapeStation System using high-sensitivity D1000 screentapes and quantified using the Qubit Fluorometer High Sensitivity dsDNA assay kit (Thermo Fisher Scientific, Q32851) Multiplexed libraries were sequenced on a NextSeq-500 Illumina platform using the NextSeq 500/550 High Output v2 kit 75 cycles (FC-404-2005).

Ribo-seq. Ribo-seq has been performed as described previously $y^{43}$ with some modifications ${ }^{32}$. Briefly, 10,000 late L4 worms were sorted using COPAS biosorter as described above and were lysed by freeze grinding in liquid nitrogen in Polysome buffer (20 mM Tris-HCl pH 8, $140 \mathrm{mM} \mathrm{KCl,} 5 \mathrm{mM} \mathrm{MgCl} 2,1 \%$ Triton X-100, $0.1 \mathrm{mg} / \mathrm{mL}$ cycloheximide) and $\sim 1 \mathrm{mg}$ extract was digested by RNase I (100 U) at $37^{\circ} \mathrm{C}$ for $5 \mathrm{~min}$. Debris was clarified by centrifuging at $18,000 \times g$ followed by fractionation on a discontinuous sucrose gradient $(10-50 \%)$ by ultracentrifugation at $260,110 \times g$ for $3 \mathrm{~h}$ in an SW41-Ti rotor (Beckman coulter). Monosome fractions were collected by pumping Fluorinert FC- 40 and using a fraction collector by measuring UV trace. RNA extracted from the monosome fraction was DNase treated as described above and fragments of 28-30 nucleotides were size selected by resolving on a $15 \%$ TBE-Urea gel. 3'phosphate was removed (PNK buffer pH 6.5 (70 mM Tris pH 6.5, $10 \mathrm{mM} \mathrm{MgCl}, 1 \mathrm{mM}$ DTT), T4 PNK (Thermo Scientific), RNaseIN 40 U/mL, 20\% PEG400) and 5'-end was phosphorylated by treating RNA with T4-Polynucleotide Kinase (1x PNK buffer (Thermo Scientific), 1 mM ATP). In all, 28-30 nucleotide Ribosome-protected fragments (RPF) were then cloned with the sRNA-seq library preparation approach, as described previously 32,66 .

Polysome profiling and blot. Lysates were fractionated on a discontinuous sucrose gradient $(10-50 \%)$ as described above, with the exception that no RNase treatment was performed. Twenty-two fractions were collected by pumping of Fluorinert FC40 and using a fraction collector while simultaneously measuring the UV trace. Fractions were precipitated with $10 \%$ TCA at $4{ }^{\circ} \mathrm{C}$ for $4 \mathrm{~h}$ and centrifuged at 
$18,000 \times g$ for $10 \mathrm{~min}$. Pellets were washed with pre-chilled acetone twice, followed by resuspension in $2 \mathrm{x}$ NuPAGE LDS sample buffer (ThermoFisher Scientific) and heated at $90^{\circ} \mathrm{C}$ for $10 \mathrm{~min}$. Samples were processed for western blotting as described above.

Immunoprecipitation-mass spectrometry (IP-MS/MS). IPs for the MS/MS analysis were performed as described previously ${ }^{66}$. Briefly, a synchronous population of 120,000 (for CSR-1 IPs for RNase treatment or control condition and PRG-1 IPs in RNase and no RNase condition) worms were harvested at $48 \mathrm{~h}$ posthatching or 20,000 (for CSR-1 IPs comparing WT IP with catalytic mutant) worms were harvested and sorted at $44 \mathrm{~h}$ post-hatching and lysed by using a chilled metal dounce in the IP buffer (50 mM HEPES pH 7.5, $300 \mathrm{mM} \mathrm{NaCl}, 5 \mathrm{mM} \mathrm{MgCl}, 10 \%$ Glycerol, $0.25 \%$ NP-40, protease inhibitor cocktails (Fermentas). Crude lysates were cleared of debris by centrifuging at $18,000 \times g$ at $4^{\circ} \mathrm{C}$ for $10 \mathrm{~min}$. For RNase treatment, RNase I (Invitrogen) $50 \mathrm{U} / \mathrm{mg}$ of the extract was used at $37^{\circ} \mathrm{C}$ for $5 \mathrm{~min}$. Approximately $5 \mathrm{mg}$ of protein extract (for CSR-1 IPs and PRG-1 IPs in RNase or control condition) or $1 \mathrm{mg}$ of protein extract (for CSR-1 IPs comparing WT IP with catalytic mutant) was incubated with $15 \mu \mathrm{l}$ of packed Anti-FLAG M2 Magnetic Agarose Beads (Sigma M8823) for $1 \mathrm{~h}$ at $4{ }^{\circ} \mathrm{C}$. After four washes with the IP buffer, the beads were washed twice with $100 \mu \mathrm{L}$ of $25 \mathrm{mM} \mathrm{NH}_{4} \mathrm{HCO}_{3}$. Finally, beads were resuspended in $100 \mu \mathrm{L}$ of $25 \mathrm{mM} \mathrm{NH}_{4} \mathrm{HCO}_{3}$ and digested by adding $0.2 \mu \mathrm{g}$ of trypsin/LysC (Promega) for $1 \mathrm{~h}$ at $37^{\circ} \mathrm{C}$. Samples were then loaded into a homemade C18 Stage Tips for desalting (principally, by stacking one $3 \mathrm{M}$ Empore SPE Extraction Disk Octadecyl (C18) and beads from SepPak C18 Cartridge Waters into a $200 \mu \mathrm{l}$ micropipette tip). Peptides were eluted using a ratio of 40:60 MeCN: $\mathrm{H}_{2} \mathrm{O}+0.1 \%$ formic acid and vacuum concentrated to dryness. Peptides were reconstituted in injection buffer (2:98 $\mathrm{MeCN}: \mathrm{H}_{2} \mathrm{O}+0.3 \%$ TFA) before nanoLC-MS/MS analysis as described previously ${ }^{66}$

\section{Data analysis}

Sequencing data analyses. Multiplexed data were demultiplexed using Illumina bcl2fastq converter version v2.17.1.14. Analysis for RNA-seq, sRNA-seq and GROseq have been performed as previously described ${ }^{32,66}$. Quality control was performed with fastQC version v0.11.5. HISAT2 version 2.0.4 was used for mapping RNA-seq data. Bowtie2 version 2.3.4.1 was used for all other sequencing data. Unless otherwise stated, computations were done using Python and UNIX utilities, either as standalone scripts or as steps implemented in a Snakemake ${ }^{71}$ workflow. The scripts and workflows are available at https://gitlab.pasteur.fr/bli/bioinfo_utils. For mapping 22G-RNA with 3' polyuridiniylation, among the small RNA reads that initially did not map, those starting with $\mathrm{G}$ followed by 20 to 25 nucleotides and then one or more Ts were selected, and their T-tail was trimmed. Those reads were then re-mapped in the same way as initially and classified using the same criteria as other small RNAs ${ }^{66}$. If classified as " $22 \mathrm{G}$ " by this procedure, they were actually considered "poly-U 22 G" or "siu 22 G".

For Ribo-seq data (data analysis pipeline available at the same address), the analysis was performed according to the following steps. The $3^{\prime}$ adapter was trimmed from raw reads using Cutadapt v.1.18 72 using the following parameter: -a TGGAATTCTCGGG TGCCAAGG -discard-untrimmed. The $5^{\prime}$ and $3^{\prime}$ UMIs were removed from the trimmed reads using cutadapt with options $-\mathrm{u} 4$ and $-\mathrm{u}-4$. After removing UMIs, the reads from 28 to $30 \mathrm{nt}$ were selected using bioawk (https://github.com/lh3/bioawk, git commit fd40150b7c557da45e781a999d372abbc634cc21).

The selected 28-30-nucleotide reads were aligned to the C. elegans genome sequence (ce11, C. elegans Sequencing Consortium WBcel235, with an added extra chromosome representing the codon-optimized klp-7 for some libraries) using Bowtie $^{73}$ v.2.3.4.3 with the following parameters: -L 6 -i S,1,0.8 -N 0 .

Reads mapping on sense orientation on annotated protein-coding genes were considered as Ribosome-protected fragments (RPF). Such reads were extracted from mapping results using samtools ${ }^{74} 1.9$ and bedtools ${ }^{75} \mathrm{v} 2.27 .1$. RPF reads of size 29 were further classified into subcategories, based on the codons found at the positions corresponding to the $\mathrm{A}(16-18 \mathrm{nt})$ and $\mathrm{P}(13-15 \mathrm{nt})$ sites of the ribosome. Codon optimality was defined as explained below. Those reads were re-mapped on the genome using bowtie2 (version 2.3.4.3) with options -L 6 -i S,1,0.8 -N 0. The resulting alignments were used to generate bigwig files with a custom bash script using bedtools version v2.27.1, bedops ${ }^{76}$ version 2.4.35, and bedGraphToBigWig version 4 . Read counts in the bigwig file were normalized by million "nonstructural" mappers, that is, reads of size 28 to $30 \mathrm{nt}$ mapping on annotation not belonging to the "structural" (tRNA, snRNA, snoRNA, rRNA, ncRNA) categories, and counted using featureCounts ${ }^{77}$ v.1.6.3. These bigwig files were used to generate "metaprofiles" where normalized coverage information (RPM for reads per million) was averaged across replicates and represented along sets of selected genes. The metaprofiles were generated using a Python script based on the deepTools ${ }^{78}$ and gffutils (https://github.com/daler/gffutils) libraries. Translation efficiency was calculated as the ratio of TPMs of Ribo-seq and RNA-seq.

Distance distribution analyses. The distribution of the distances between re-mapped RPF and 22G-RNA-seq reads was computed by counting distances between 5 '-ends of RPF and 22G-RNA reads of opposite strandedness, only considering 22G-RNA reads within a distance of $+/-120 \mathrm{bp}$ from the RPF read and only considering RPF reads mapping in the sense direction within the coordinates of a gene among a selected list. Counts were transformed into $\mathrm{z}$-scores using the Scipy ${ }^{79}$ library (version 1.3.2). A plot of distance distribution, within the $(-15,45)$ distance range, was made using the Matplotlib library (https://doi.org/10.1109/MCSE.2007.55) version 3.1.1. This was done using $z$-scores in order to have comparable values between different combinations of libraries. A plot of dominant periods in distance distribution signal was made using the Matplotlib library (https://doi.org/10.1109/MCSE.2007.55) version 3.1.1. The dominant periods were obtained using the fast Fourier transform function of the Scipy library (version 1.3.2) ${ }^{79}$. This was done using $z$-scores in order to have comparable values between different combinations of libraries.

Read composition analyses. Reads of a class of interest (small RNAs, or sizerestricted subclasses thereof, RPF or RPF subclasses) and found mapping on a given gene, either on the CDS or on the first $100 \mathrm{bp}$ of the 3 UTR and either mapping in sense or antisense with respect to the gene annotation, were gathered from their remapping results using custom Python code based on pysam (https://github.com/ pysam-developers/pysam) a Python wrapper for the samtools package ${ }^{74}$. For a given such set of reads, the proportions of each nucleotide at each position starting from the 5'end or from the 3 '-end of the reads were computed. These proportions were averaged across a set of genes of interest and represented as a stacked barplot, where each stack corresponds to a position in the reads, with the most frequent nucleotides on top. For comparing read composition of sRNA from CDS and 3'UTR, to account for overall genome composition variability across genes or gene features, "scaled" proportions were computed by dividing the nucleotide proportions by those found in the genomic region on which the reads were found mapping (CDS or first $100 \mathrm{bp}$ of 3 UTR of a given gene), then similarly averaged across a set of genes of interest and represented as a stacked barplot. The core functionalities used in these analyses are implemented in the following Python library: https://gitlab.pasteur.fr/bli/ libreads. The whole code we used is available upon request.

Analysis of codon usage. All protein-coding genes were categorized based on their Translation Efficiency in the following categories $\log _{2} \mathrm{TE} \geq 3, \geq 2, \geq 1, \leq-1, \leq-2$, and $\leq-3$. Relative synonymous codon usage was calculated for genes in each category using the CAI calculator ${ }^{80}$. To calculate enrichment of codons usage in each of the categories, differential RSCU of respective categories of genes was calculated by normalizing their RSCU with RSCU of genes showing a TE of $\sim 1$ $\left(\log _{2} \mathrm{TE} 0 \pm 0.1\right)$. Codons enriched in highly translated mRNAs $\left(\log _{2} \mathrm{TE} \geq 3\right)$ were considered optimal codons, and codons that were avoided were considered nonoptimal. Similarly, differential RSCU analysis was performed for CSR-1 targets.

Gene ontology and enrichment analysis. Gene ontology was performed using WormCat tool ${ }^{81}$. Enrichment was calculated using the webtool http://nemates.org/ MA/progs/overlap_stats.html.

tRNA copy number and TPM. tRNA copy number was determined using tRNAscan-SE 2.082. TPMs for the tRNAs were extracted from the GRO-seq dataset from WT late L4-staged worms.

Determination of a codon-optimized sequence for klp-7. The codon-optimized sequence for $k l p-7$ was computed with a Python script using BioPython ${ }^{83}$ as follows: To each amino acid, a corresponding optimal codon was associated based on a given optimality ranking. Here, the codon ranking was based on usage in highly translation efficient proteins, as explained above. Then, each codon in the CDS of the native $k l p-7$ gene was replaced with the optimal codon associated with the corresponding amino acid. For mapping purposes, the resulting sequence was added to the genome as if it was an extra chromosome, and the transgene was added to the annotation files used for read counting. In order to produce comparable bigwig tracks between libraries obtained on different strains (codon-optimized or not), a Python script based on the pyBigWig library (https://doi.org/ 10.5281/zenodo.594045) was used to relocate the values on the extra chromosome to the actual genomic position of $k l p-7$.

MS/MS data analysis. For identification, the data were searched against the C. elegans (CAEEL) UP000001940 database (Taxonomy 6239 containing one protein sequence par gene) using Sequest HT through Proteome Discoverer (v.2.2). Enzyme specificity was set to trypsin, and a maximum of two missed cleavage sites was allowed. Oxidized methionine and $\mathrm{N}$-terminal acetylation were set as variable modifications. Maximum allowed mass deviation was set to $10 \mathrm{ppm}$ for monoisotopic precursor ions and 0.6 Da for MS/MS peaks. The resulting files were further processed using myProMS v.3.9 $9^{84}$ (work in progress). False-discovery rate (FDR) was calculated using Percolator and was set to $1 \%$ at the peptide level for the whole study. Label-free quantification was performed using peptide extracted ion chromatograms (XICs), computed with MassChroQ v.2.2.185. For protein quantification, XICs from proteotypic peptides shared between compared conditions (TopN matching) with missed cleavages were used. Median and scale normalization was applied on the total signal to correct the XICs for each biological replicate $(N=4)$. To estimate the significance of the change in protein abundance, a statistical test based on a linear model adjusted on peptides and biological replicates was performed, and $P$-values were adjusted using the

Benjamini-Hochberg FDR. Proteins with at least three total peptides in all replicates, a twofold enrichment, and an adjusted $P$-value $<0.05$ were considered 
significantly enriched in sample comparisons. The MS proteomics data have been deposited to the ProteomeXchange Consortium via the PRIDE ${ }^{86}$ partner repository with the dataset identifier PXD012557 and PXD020293.

Statistics and reproducibility. Almost all the experiments shown in this study were performed independently at least twice, and no inconsistent results were observed. IP and MS experiments were conducted with four biological replicates. Ribo-seq was performed using three biological replicates. All the RNA-seq experiments, GRO-seq, sRNA-seq, IP-sRNA-seq, were performed using two biological replicates. RT-qPCRs to test RNAi efficiency in samples for sequencing experiments were performed in their respective biological experiments. RT-qPCRs for gene expression changes otherwise were performed with at least three biological replicates. Most of the graphs were generated using GraphPad Prism 9. Log fold-changes for almost all the plots were calculated on the mean of biologically independent replicates. For details of the particular statistical analyses used, precise $P$-values, statistical significance, and sample sizes for all of the graphs, see the figure legends.

Gene lists. The gene lists generated in this studyare provided in Supplementary Data 1 together with previously identified gene lists ${ }^{14,22,23,25,66,87,88}$.

Reporting summary. Further information on research design is available in the Nature Research Reporting Summary linked to this article.

\section{Data availability}

All sequencing data (GRO-seq, RNA-seq, and sRNA-seq from total lysate or IP experiments, Ribo-seq) are available at the Gene Expression Omnibus (GEO) under accession code GSE155077. The MS proteomics data have been deposited to the ProteomeXchange Consortium via the PRIDE partner repository with the dataset identifier PXD012557 and PXD020293. The data supporting the findings of this study are available from the corresponding authors upon reasonable request. Source data are provided with this paper.

\section{Code availability}

Custom scripts of this study are available from the corresponding author on request. Custom code and data analysis workflows are available at https://gitlab.pasteur.fr/bli/ bioinfo_utils.

Received: 4 October 2020; Accepted: 6 May 2021;

Published online: 09 June 2021

\section{References}

1. Ghildiyal, M. \& Zamore, P. D. Small silencing RNAs: an expanding universe. Nat. Rev. Genet. 10, 94-108 (2009).

2. Okamura, K. \& Lai, E. C. Endogenous small interfering RNAs in animals. Nat. Rev. Mol. Cell Biol. 9, 673-678 (2008).

3. Hammond, S. M., Bernstein, E., Beach, D. \& Hannon, G. J. An RNA-directed nuclease mediates post-transcriptional gene silencing in Drosophila cells. Nature 404, 293-296 (2000).

4. Williams, R. W. \& Rubin, G. M. ARGONAUTE1 is required for efficient RNA interference in Drosophila embryos. Proc. Natl Acad. Sci. USA 99, 6889-6894 (2002).

5. Tabara, H. et al. The rde-1 gene, RNA interference, and transposon silencing in C. elegans. Cell 99, 123-132 (1999).

6. Grishok, A. et al. Genes and mechanisms related to RNA interference regulate expression of the small temporal rnas that control C. elegans developmental timing. Cell 106, 23-34 (2001).

7. Billi, A. C., Fischer, S. E. J. \& Kim, J. K. Endogenous RNAi pathways in C. elegans. Wormbook 1-49, https://doi.org/10.1895/wormbook.1.170.1 (2014)

8. Das, P. P. et al. Piwi and piRNAs act upstream of an endogenous siRNA pathway to suppress Tc3 transposon mobility in the caenorhabditis elegans germline. Mol. Cell 31, 79-90 (2008).

9. Bagijn, M. P. et al. Function, targets, and evolution of Caenorhabditis elegans piRNAs. Science 337, 574-578 (2012).

10. Batista, P. J. et al. PRG-1 and 21U-RNAs interact to form the piRNA complex required for fertility in C. elegans. Mol. Cell 31, 67-78 (2008)

11. Lee, H.-C. et al. C. elegans piRNAs mediate the genome-wide surveillance of germline transcripts. Cell 150, 78-87 (2012).

12. Cecere, G., Zheng, G. X. Y., Mansisidor, A. R., Klymko, K. E. \& Grishok, A. Promoters recognized by forkhead proteins exist for individual 21U-RNAs. Mol. Cell 47, 734-745 (2012).

13. Maniar, J. M. \& Fire, A. Z. EGO-1, a C. elegans RdRP, modulates gene expression via production of mRNA-templated short antisense RNAs. Curr. Biol. 21, 449-459 (2011).
14. Claycomb, J. M. et al. The argonaute CSR-1 and Its 22G-RNA cofactors are required for holocentric chromosome segregation. Cell 139, 123-134 (2009).

15. Seth, $M$. et al. The coding regions of germline mRNAs confer sensitivity to argonaute regulation in C. elegans. Cell Rep. 22, 2254-2264 (2018).

16. Shen, E.-Z. et al. Identification of piRNA binding sites reveals the argonaute regulatory landscape of the C. elegans germline. Cell 172, 937-951 (2018). e18

17. Zhang, D. et al. The piRNA targeting rules and the resistance to piRNA silencing in endogenous genes. Science 359, 587-592 (2018).

18. Seth, M. et al. The C. elegans CSR-1 argonaute pathway counteracts epigenetic silencing to promote germline gene expression. Dev. Cell 27, 656-663 (2013).

19. Wedeles, C. J., Wu, M. Z. \& Claycomb, J. M. Protection of germline gene expression by the C. elegans argonaute CSR-1. Dev. Cell 27, 664-671 (2013).

20. Tyc, K. M. et al. The conserved intron binding protein EMB-4 plays differential roles in germline small RNA pathways of C. elegans. Dev. Cell 42, 256-270 (2017). e6.

21. Akay, A. et al. The helicase aquarius/EMB-4 is required to overcome intronic barriers to allow nuclear RNAi pathways to heritably silence transcription. Dev. Cell 42, 241-255 (2017). e6.

22. Conine, C. C. et al. Argonautes promote male fertility and provide a paternal memory of germline gene expression in C. elegans. Cell 155, 1532-1544 (2013).

23. Cecere, G., Hoersch, S., O’Keeffe, S., Sachidanandam, R. \& Grishok, A. Global effects of the CSR-1 RNA interference pathway on the transcriptional landscape. Nat. Struct. Mol. Biol. 21, nsmb.2801 (2014).

24. Aoki, K., Moriguchi, H., Yoshioka, T., Okawa, K. \& Tabara, H. In vitro analyses of the production and activity of secondary small interfering RNAs in C. elegans. EMBO J. 26, 5007-5019 (2007).

25. Gerson-Gurwitz, A. et al. A small RNA-catalytic argonaute pathway tunes germline transcript levels to ensure embryonic divisions. Cell 165, 396-409 (2016).

26. Gu, W. et al. Distinct argonaute-mediated 22G-RNA pathways direct genome surveillance in the C. elegans germline. Mol. Cell 36, 231-244 (2009).

27. Wan, G. et al. Spatiotemporal regulation of liquid-like condensates in epigenetic inheritance. Nature 557, 679-683 (2018).

28. Phillips, C. M., Montgomery, T. A., Breen, P. C. \& Ruvkun, G. MUT-16 promotes formation of perinuclear mutator foci required for RNA silencing in the C. elegans germline. Gene Dev. 26, 1433-1444 (2012).

29. Zhang, C. et al. mut-16 and other mutator class genes modulate $22 \mathrm{G}$ and $26 \mathrm{G}$ siRNA pathways in Caenorhabditis elegans. Proc. Natl Acad. Sci. USA 108 1201-1208 (2011)

30. Preston, M. A. et al. Unbiased screen of RNA tailing activities reveals a poly (UG) polymerase. Nat. Methods 16, 437-445 (2019)

31. Shukla, A. et al. poly(UG)-tailed RNAs in genome protection and epigenetic inheritance. Nature 1-6, https://doi.org/10.1038/s41586-020-2323-8 (2020).

32. Quarato, P. et al. Germline inherited small RNAs facilitate the clearance of untranslated maternal mRNAs in C. elegans embryos. Nat. Commun. 12, 1441 (2021).

33. Yigit, E. et al. Analysis of the C. elegans argonaute family reveals that distinct argonautes act sequentially during RNAi. Cell 127, 747-757 (2006).

34. Conine, C. C. et al. Argonautes ALG-3 and ALG-4 are required for spermatogenesis-specific 26G-RNAs and thermotolerant sperm in Caenorhabditis elegans. Proc. Natl Acad. Sci. USA 107, 3588-3593 (2010).

35. Han, T. et al. 26G endo-siRNAs regulate spermatogenic and zygotic gene expression in Caenorhabditis elegans. Proc. Natl Acad. Sci. USA 106, 18674-18679 (2009).

36. van Wolfswinkel, J. C. et al. CDE-1 affects chromosome segregation through uridylation of CSR-1-bound siRNAs. Cell 139, 135-148 (2009).

37. Kim, J. K. et al. Functional genomic analysis of RNA interference in C. elegans. Science 308, 1164-1167 (2005).

38. Tops, B. B. J. et al. RDE-2 interacts with MUT-7 to mediate RNA interference in Caenorhabditis elegans. Nucleic Acids Res. 33, 347-355 (2005).

39. Chen, C.-C. G. et al. A member of the polymerase $\beta$ nucleotidyltransferase superfamily is required for RNA interference in C. elegans. Curr. Biol. 15 , 378-383 (2005)

40. Updike, D. L., Knutson, A. K., Egelhofer, T. A., Campbell, A. C. \& Strome, S. Germ-granule components prevent somatic development in the C. elegans germline. Curr. Biol. 24, 970-975 (2014).

41. Campbell, A. C. \& Updike, D. L. CSR-1 and P granules suppress spermspecific transcription in the C. elegans germline. Development 142, 1745-1755 (2015).

42. Lee, C.-Y. S. et al. Recruitment of mRNAs to P granules by condensation with intrinsically-disordered proteins. Elife 9, e52896 (2020).

43. Aeschimann, F., Xiong, J., Arnold, A., Dieterich, C. \& Großhans, H. Transcriptome-wide measurement of ribosomal occupancy by ribosome profiling. Methods 85, 75-89 (2015).

44. Schisa, J. A., Pitt, J. N. \& Priess, J. R. Analysis of RNA associated with P granules in germ cells of C. elegans adults. Dev. Camb. Engl. 128, 1287-1298 (2001). 
45. Pechmann, S. \& Frydman, J. Evolutionary conservation of codon optimality reveals hidden signatures of cotranslational folding. Nat. Struct. Mol. Biol. 20, 237-243 (2012).

46. Presnyak, V. et al. Codon optimality is a major determinant of mRNA stability. Cell 160, 1111-1124 (2015).

47. Stadler, M. \& Fire, A. Wobble base-pairing slows in vivo translation elongation in metazoans. RNA 17, 2063-2073 (2011).

48. Duret, L. tRNA gene number and codon usage in the C. elegans genome are co-adapted for optimal translation of highly expressed genes. Trends Genet. 16, 287-289 (2000).

49. Novoa, E. M. \& de Pouplana, L. R. Speeding with control: codon usage, tRNAs, and ribosomes. Trends Genet. 28, 574-581 (2012).

50. Tuller, T. et al. An evolutionarily conserved mechanism for controlling the efficiency of protein translation. Cell 141, 344-354 (2010).

51. Gigant, E. et al. Inhibition of ectopic microtubule assembly by the kinesin-13 KLP-7MCAK prevents chromosome segregation and cytokinesis defects in oocytes. Dev. Camb. Engl. 144, 1674-1686 (2017).

52. Maine, E. M. et al. EGO-1, a putative RNA-dependent RNA polymerase, is required for heterochromatin assembly on unpaired DNA during C. elegans meiosis. Curr. Biol. 15, 1972-1978 (2005).

53. Gushchanskaia, E. S., Esse, R., Ma, Q., Lau, N. C. \& Grishok, A. Interplay between small RNA pathways shapes chromatin landscapes in C. elegans. Nucleic Acids Res. 47, 5603-5616 (2019).

54. Hubstenberger, A. et al. P-body purification reveals the condensation of repressed mRNA regulons. Mol. Cell 68, 144-157 (2017). e5.

55. Pelechano, V., Wei, W. \& Steinmetz, L. M. Widespread co-translational RNA decay reveals ribosome dynamics. Cell 161, 1400-1412 (2015).

56. Zinoviev, A., Ayupov, R. K., Abaeva, I. S., Hellen, C. U. T. \& Pestova, T. V. Extraction of mRNA from stalled ribosomes by the Ski complex. Mol. Cell 77, 1340-1349 (2020). e6.

57. Ibrahim, F., Maragkakis, M., Alexiou, P. \& Mourelatos, Z. Ribothrypsis, a novel process of canonical mRNA decay, mediates ribosome-phased mRNA endonucleolysis. Nat. Struct. Mol. Biol. 25, 302-310 (2018).

58. Ruijtenberg, S. et al. mRNA structural dynamics shape Argonaute-target interactions. Nat. Struct. Mol. Biol. 27, 790-801 (2020).

59. Małecka, E. M. \& Woodson, S. A. Ribosomes clear the way for siRNA targeting. Nat. Struct. Mol. Biol. 27, 775-777 (2020).

60. Pule, M. N., Glover, M. L., Fire, A. Z. \& Arribere, J. A. Ribosome clearance during RNA interference. RNA 25, rna.070813.119 (2019).

61. Sun, Y. et al. Ribosomes guide pachytene piRNA formation on long intergenic piRNA precursors. Nat. Cell Biol. 22, 200-212 (2020).

62. $\mathrm{Wu}, \mathrm{H}$. et al. Plant 22-nt siRNAs mediate translational repression and stress adaptation. Nature 1-5, https://doi.org/10.1038/s41586-020-2231-y (2020).

63. Iwakawa, $\mathrm{H}$. et al. Ribosome stalling caused by the Argonaute-miRNA-SGS3 complex regulates production of secondary siRNA biogenesis in plants. Preprint at bioRxiv, https://doi.org/10.1101/2020.09.10.288902 (2020).

64. Brenner, S. The genetics of Caenorhabditis elegans. Genetics 77, 71-94 (1974).

65. Paix, A., Folkmann, A., Rasoloson, D. \& Seydoux, G. High efficiency, homology-directed genome editing in Caenorhabditis elegans Using CRISPRCas9 ribonucleoprotein complexes. Genetics 201, 47-54 (2015).

66. Barucci, G. et al. Small-RNA-mediated transgenerational silencing of histone genes impairs fertility in piRNA mutants. Nat. Cell Biol. 22, 1-11 (2020).

67. Dokshin, G. A., Ghanta, K. S., Piscopo, K. M. \& Mello, C. C. Robust genome editing with short single-stranded and long, partially single-stranded DNA donors in Caenorhabditis elegans. Genetics 210, 781-787 (2018).

68. Kamath, R. S. \& Ahringer, J. Genome-wide RNAi screening in Caenorhabditis elegans. Methods 30, 313-321 (2003).

69. Kawasaki, I. et al. PGL-1, a predicted RNA-binding component of germ granules, is essential for fertility in C. elegans. Cell 94, 635-645 (1998).

70. Simon, M. et al. Reduced insulin/IGF-1 signaling restores germ cell immortality to Caenorhabditis elegans Piwi mutants. Cell Rep. 7, 762-773 (2014).

71. Koster, J. \& Rahmann, S. Snakemake-a scalable bioinformatics workflow engine. Bioinformatics 28, 2520-2522 (2012).

72. Martin, M. Cutadapt removes adapter sequences from high-throughput sequencing reads. Embnet J. 17, 10-12 (2011).

73. Langmead, B. \& Salzberg, S. L. Fast gapped-read alignment with Bowtie 2. Nat. Methods 9, 357-359 (2012).

74. $\mathrm{Li}, \mathrm{H}$. et al. The sequence alignment/map format and SAMtools. Bioinformatics 25, 2078-2079 (2009).

75. Quinlan, A. R. \& Hall, I. M. BEDTools: a flexible suite of utilities for comparing genomic features. Bioinformatics 26, 841-842 (2010).

76. Neph, S. et al. BEDOPS: high-performance genomic feature operations. Bioinformatics 28, 1919-1920 (2012).

77. Liao, Y., Smyth, G. K. \& Shi, W. featureCounts: an efficient general purpose program for assigning sequence reads to genomic features. Bioinformatics 30, 923-930 (2013).
78. Ramírez, F. et al. deepTools2: a next generation web server for deepsequencing data analysis. Nucleic Acids Res. 44, W160-W165 (2016).

79. Virtanen, P. et al. SciPy 1.0: fundamental algorithms for scientific computing in Python. Nat. Methods 17, 261-272 (2020).

80. Puigbò, P., Bravo, I. G. \& Garcia-Vallve, S. CAIcal: A combined set of tools to assess codon usage adaptation. Biol. Direct 3, 38 (2008).

81. Holdorf, A. D. et al. WormCat: an online tool for annotation and visualization of Caenorhabditis elegans genome-scale data. Genetics 214, 279-294 (2019).

82. Chan, P. P. \& Lowe, T. M. Gene prediction, methods and protocols. Methods Mol. Biol. Clifton N. J. 1962, 1-14 (2019).

83. Cock, P. J. A. et al. Biopython: freely available Python tools for computationa molecular biology and bioinformatics. Bioinformatics 25, 1422-1423 (2009).

84. Poullet, P., Carpentier, S. \& Barillot, E. myProMS, a web server for management and validation of mass spectrometry-based proteomic data. Proteomics 7, 2553-2556 (2007).

85. Valot, B., Langella, O., Nano, E. \& Zivy, M. MassChroQ: a versatile tool for mass spectrometry quantification. Proteomics 11, 3572-3577 (2011).

86. Vizcaíno, J. A. et al. 2016 update of the PRIDE database and its related tools. Nucleic Acids Res. 44, 11033-11033 (2016).

87. Ortiz, M. A., Noble, D., Sorokin, E. P. \& Kimble, J. A new dataset of spermatogenic vs. oogenic transcriptomes in the nematode Caenorhabditis elegans. G3 Genes Genomes Genet. 4, g3.114.012351 (2014).

88. Reed, K. J. et al. Widespread roles for piRNAs and WAGO-class siRNAs in shaping the germline transcriptome of Caenorhabditis elegans. Nucleic Acids Res. 48, 1811-1827 (2019).

89. Love, M. I., Huber, W. \& Anders, S. Moderated estimation of fold change and dispersion for RNA-seq data with DESeq2. Genome Biol. 15, 550 (2014).

90. Reis, M., dos, Savva, R. \& Wernisch, L. Solving the riddle of codon usage preferences: a test for translational selection. Nucleic Acids Res. 32, 5036-5044 (2004).

\section{Acknowledgements}

We would like to thank all the members of the Cecere laboratory, Manish Grover, Sudarshan Gadadhar, and Angela Anderson (Life Science Editors), for the helpful discussions on the paper. We thank Micheline Fromont for her help to set up Ribosome profiling. We thank Celine Didier for technical assistance. Sequencing was performed at the Biomics centre at the Institut Pasteur. We thank the Heng-Chi Lee lab, Miska lab, Desai lab, Strome lab, Mello lab, Updike lab, and Kennedy lab for sharing strains and reagents. Some strains were provided by the CGC, funded by the NIH Office of Research Infrastructure Programs (P40 OD010440). This project has received funding from the Institut Pasteur, the CNRS, and the European Research Council (ERC) under the European Union's Horizon 2020 research and innovation program under grant agreement No. ERC-StG- 679243. M.S. and E.C. were supported by the Pasteur-Roux-Cantarini Postdoctoral Fellowship program. P.Q. was supported by Ligue Nationale Contre le Cancer (SFB19032). F.D. and D.L. have received funding from Région Ile-de-France and Fondation pour la Recherche Médicale grants to support this study.

\section{Author contributions}

G.C. and M.S. identified and developed the core questions addressed in the project. M.S. performed most of the experiments and analyzed the results together with G.C. E.C. and L.B. generated the strains used in this study with the help of M.S. and P.Q. E.C. performed the RNA-seq for CSR-1 mutants and IP-sRNA-seq of HRDE-1 in CSR-1 KO and phenotypic characterization of csr-1 mutants. P.Q. performed GRO-seq for CSR-1 mutants. B.L. performed all the bioinformatics analysis along with M.S. S.P. contributed for distance mapping analysis of 22G-RNA reads, and Ribo-seq reads with B.L. F.D. and D.L. performed MS/MS experiments and analyzed the data together with M.S. M.S. and G.C. wrote the paper with the contribution of all authors.

\section{Competing interests}

The authors declare no competing interests.

\section{Additional information}

Supplementary information The online version contains supplementary material available at https://doi.org/10.1038/s41467-021-23615-w.

Correspondence and requests for materials should be addressed to G.C.

Peer review information Nature Communications thanks Zissimos Mourelatos and other, anonymous, reviewers for their contribtuions to the peer review of this work. Peer review reports are available.

Reprints and permission information is available at http://www.nature.com/reprints

Publisher's note Springer Nature remains neutral with regard to jurisdictional claims in published maps and institutional affiliations. 
(c) (i) Open Access This article is licensed under a Creative Commons Attribution 4.0 International License, which permits use, sharing, adaptation, distribution and reproduction in any medium or format, as long as you give appropriate credit to the original author(s) and the source, provide a link to the Creative Commons license, and indicate if changes were made. The images or other third party material in this article are included in the article's Creative Commons license, unless indicated otherwise in a credit line to the material. If material is not included in the article's Creative Commons license and your intended use is not permitted by statutory regulation or exceeds the permitted use, you will need to obtain permission directly from the copyright holder. To view a copy of this license, visit http://creativecommons.org/ licenses/by/4.0/.

(C) The Author(s) 2021 